MATHEMATICS OF COMPUTATION

Volume 69, Number 232, Pages 1385-1407

S 0025-5718(00)01248-5

Article electronically published on March 24, 2000

\title{
CONVERGENCE OF GAUGE METHOD FOR INCOMPRESSIBLE FLOW
}

\author{
CHENG WANG AND JIAN-GUO LIU
}

\begin{abstract}
A new formulation, a gauge formulation of the incompressible Navier-Stokes equations in terms of an auxiliary field $\mathbf{a}$ and a gauge variable $\phi, \mathbf{u}=\mathbf{a}+\nabla \phi$, was proposed recently by $\mathrm{E}$ and Liu. This paper provides a theoretical analysis of their formulation and verifies the computational advantages. We discuss the implicit gauge method, which uses backward Euler or Crank-Nicolson in time discretization. However, the boundary conditions for the auxiliary field a are implemented explicitly (vertical extrapolation). The resulting momentum equation is decoupled from the kinematic equation, and the computational cost is reduced to solving a standard heat and Poisson equation. Moreover, such explicit boundary conditions for the auxiliary field a will be shown to be unconditionally stable for Stokes equations. For the full nonlinear Navier-Stokes equations the time stepping constraint is reduced to the standard CFL constraint $\triangle t / \triangle x \leq C$. We also prove first order convergence of the gauge method when we use MAC grids as our spatial discretization.

The optimal error estimate for the velocity field is also obtained.
\end{abstract}

\section{IntRoduction AND REVIEW OF THE GAUGE METHOD}

We start with the homogeneous, incompressible Navier-Stokes equations (NSE) with no-slip boundary condition:

$$
\left\{\begin{array}{l}
\boldsymbol{u}_{t}+(\boldsymbol{u} \cdot \nabla) \boldsymbol{u}+\nabla p=\frac{1}{R e} \Delta \boldsymbol{u}, \quad \text { in } \Omega, \\
\nabla \cdot \boldsymbol{u}=0, \quad \text { in } \Omega, \\
\boldsymbol{u}=0, \quad \text { on } \partial \Omega,
\end{array}\right.
$$

where $\boldsymbol{u}$ is the velocity, $p$ is the pressure and Re is the Reynolds number.

A new gauge formulation was proposed by $\mathrm{E}$ and Liu in [6]. Instead of using primitive variables of NSE, the gauge method replaces pressure by a gauge variable $\phi$ and introduces the auxiliary field $\boldsymbol{a}=\boldsymbol{u}-\nabla \phi$. Then the incompressibility constraint in (1.1) becomes

$$
\triangle \phi=-\nabla \cdot \boldsymbol{a}
$$

Received by the editor November 10, 1997 and, in revised form, December 7, 1998.

1991 Mathematics Subject Classification. Primary 65M12, 76M20.

Key words and phrases. Viscous incompressible flows, gauge method, convergence, explicit boundary condition.

The research was supported by NSF grant DMS-9805621 and Navy ONR grant N00014-961013.

(C)2000 American Mathematical Society 
and the momentum equation in (1.1) becomes

$$
\boldsymbol{a}_{t}+(\boldsymbol{u} \cdot \nabla) \boldsymbol{u}+\nabla\left(\partial_{t} \phi-\frac{1}{R e} \triangle \phi+p\right)=\frac{1}{R e} \triangle \boldsymbol{a} .
$$

If we require that

$$
\partial_{t} \phi-\frac{1}{R e} \triangle \phi=-p,
$$

we obtain the gauge formulation of NSE:

$$
\left\{\begin{array}{l}
\boldsymbol{a}_{t}+(\boldsymbol{u} \cdot \nabla) \boldsymbol{u}=\frac{1}{R e} \triangle \boldsymbol{a}, \quad \text { in } \Omega, \\
\triangle \phi=-\nabla \cdot \boldsymbol{a}, \quad \text { in } \Omega, \\
\boldsymbol{u}=\boldsymbol{a}+\nabla \phi, \quad \text { in } \Omega .
\end{array}\right.
$$

One of the main advantages of gauge formulation is that $\phi$ is a non-physical variable, so we have the freedom to assign boundary condition for $\phi$. As pointed out in [6], corresponding to the no-slip boundary condition $\boldsymbol{u}=0$ on $\partial \Omega$, we can prescribe either

$$
\frac{\partial \phi}{\partial \boldsymbol{n}}=0, \quad \boldsymbol{a} \cdot \boldsymbol{n}=0, \quad \boldsymbol{a} \cdot \boldsymbol{\tau}=-\frac{\partial \phi}{\partial \boldsymbol{\tau}}, \quad \text { on } \partial \Omega,
$$

or

$$
\phi=0, \quad \boldsymbol{a} \cdot \boldsymbol{n}=-\frac{\partial \phi}{\partial \boldsymbol{n}}, \quad \boldsymbol{a} \cdot \boldsymbol{\tau}=0, \quad \text { on } \partial \Omega,
$$

where $\boldsymbol{n}$ is the normal vector and $\boldsymbol{\tau}$ is the unit tangent vector. The system (1.5), (1.6) is called the Neumann gauge formulation and (1.5), (1.7) is called the Dirichlet gauge formulation. In this paper, we will concentrate on the Neumann formulation, and only give a brief description of the analysis with respect to the Dirichlet formulation.

The idea of gauge formulation has a long history. For example, Oseledets first used an impulse variable to reformulate Euler equations as in a Hamiltonian system in [15]; Buttke first used an impulse variable as a computational method in 4]; Maddocks and Pego used an impulse variable to formulate an unconstrained Hamiltonian for the Euler equation in [13. In 9], E and Liu found that the velicity impulse formulation of Buttke [4] is marginally ill-posed for the inviscid flow, and presented numerical evidence of this instability. In [16, Russo and Smereka studied the connection between different impulse/gauge formulations, especially the stretching effects.

We can write the Neumann gauge formulation (1.5) and (1.6) in another form:

$$
\begin{gathered}
\left\{\begin{array}{l}
\boldsymbol{a}_{t}+(\boldsymbol{u} \cdot \nabla) \boldsymbol{u}=\frac{1}{R e} \triangle \boldsymbol{a}, \quad \text { in } \Omega, \\
\boldsymbol{a} \cdot \boldsymbol{n}=0, \quad \boldsymbol{a} \cdot \boldsymbol{\tau}=-\frac{\partial \phi}{\partial \boldsymbol{\tau}}, \quad \text { on } \partial \Omega,
\end{array}\right. \\
\left\{\begin{array}{l}
\triangle \phi=-\nabla \cdot \boldsymbol{a}, \quad \text { in } \Omega, \\
\frac{\partial \phi}{\partial \boldsymbol{n}}=0,
\end{array} \quad \text { on } \partial \Omega .\right.
\end{gathered}
$$

With this new formulation at hand, we can easily solve (1.8) by finite difference [6], finite element [7], or other kinds of numerical techniques such as spectral element 
methods [11. We only consider finite difference here. In this paper, we are mainly concerned with the case when the Reynolds number is $\mathrm{O}(1)$, which requires us to treat the diffusion term implicitly. For example, if the backward Euler method is used as our time discretization for the momentum equation, we have

$$
\frac{\boldsymbol{a}^{n+1}-\boldsymbol{a}^{n}}{\triangle t}+\left(\boldsymbol{u}^{n} \cdot \nabla\right) \boldsymbol{u}^{n}=\triangle \boldsymbol{a}^{n+1}, \quad \text { in } \Omega .
$$

For simplicity in this presentation, we have taken $R e=1$ in (1.9). It is evident that the implementation of (1.9) requires that the boundary conditions for $\boldsymbol{a}$ be determined. To avoid the coupling between the momentum equation and the boundary conditions, we use explicit boundary conditions for $\boldsymbol{a}$, which are carried out by vertical extrapolation. For the first order scheme, we can just take

$$
\boldsymbol{a}^{n+1} \cdot \boldsymbol{n}=0, \quad \boldsymbol{a}^{n+1} \cdot \boldsymbol{\tau}=-\frac{\partial \phi^{n}}{\partial \boldsymbol{\tau}}, \quad \text { on } \partial \Omega .
$$

Next we update $\phi^{n+1}$ at time step $t^{n+1}$ by

$$
\left\{\begin{array}{l}
\triangle \phi^{n+1}=-\nabla \cdot \boldsymbol{a}^{n+1}, \quad \text { in } \Omega, \\
\frac{\partial \phi^{n+1}}{\partial \boldsymbol{n}}=0, \quad \text { on } \partial \Omega,
\end{array}\right.
$$

and the velocity $\boldsymbol{u}^{n+1}$ is determined by the incompressiblity

$$
\boldsymbol{u}^{n+1}=\boldsymbol{a}^{n+1}+\nabla \phi^{n+1} .
$$

We emphasize that the momentum equation (1.9) is decoupled from the kinematic equation (1.11), due to the fact that the boundary conditions for $\boldsymbol{a}$ in (1.10) are explicit. The resulting scheme is very efficient, and the computational cost is reduced to solving a standard heat and Poisson equation. As reported in [6], full accuracy was obtained with this explicit boundary condition.

1.1. Stability of the explicit boundary condition. One of the main concerns in computations is the stability of the scheme. The main observation of this paper is that the explicit boundary conditions (1.10) are unconditionally stable for Stokes equations, where nonlinear terms are neglected. Using the method mentioned above, we can write our scheme as

$$
\left\{\begin{array}{l}
\frac{\boldsymbol{a}^{n+1}-\boldsymbol{a}^{n}}{\triangle t}=\triangle \boldsymbol{a}^{n+1}, \quad \text { in } \Omega, \\
\boldsymbol{a}^{n+1} \cdot \boldsymbol{n}=0, \quad \boldsymbol{a}^{n+1} \cdot \boldsymbol{\tau}=-\frac{\partial \phi^{n}}{\partial \boldsymbol{\tau}}, \quad \text { on } \partial \Omega
\end{array}\right.
$$

then we obtain $\phi^{n+1}$ via (1.11), and finally, the velocity is given by (1.12).

For the convenience of our analysis below, we introduce $\hat{\boldsymbol{u}}^{n}=\boldsymbol{a}^{n+1}+\nabla \phi^{n}$. The system (1.13), (1.11), (1.12) can be reformulated as

$$
\left\{\begin{array}{l}
\frac{\hat{\boldsymbol{u}}^{n}-\boldsymbol{u}^{n}}{\Delta t}+\Delta \nabla \phi^{n}=\Delta \hat{\boldsymbol{u}}^{n}, \quad \text { in } \Omega, \\
\hat{\boldsymbol{u}}^{n}=0, \quad \text { on } \partial \Omega,
\end{array}\right.
$$




$$
\begin{cases}\boldsymbol{u}^{n+1}-\hat{\boldsymbol{u}}^{n}+\nabla\left(\phi^{n}-\phi^{n+1}\right)=0, & \text { in } \Omega, \\ \nabla \cdot \boldsymbol{u}^{n+1}=0, \quad \text { in } \Omega, & \\ \frac{\partial\left(\phi^{n}-\phi^{n+1}\right)}{\partial \boldsymbol{n}}=\boldsymbol{n} \cdot \boldsymbol{u}^{n+1}=0, & \text { on } \partial \Omega .\end{cases}
$$

This formulation is similar to the pressure increment formulation of the second order projection method in [3, 21]. So we can apply techniques similar to those used in [8] to analyze the stability of the system (1.14).

The basic technique used here is just a standard energy estimate. As can be seen, if we take the inner product of the equation in (1.14a) with $2 \hat{\boldsymbol{u}}^{n}$, and use the boundary conditions for $\hat{\boldsymbol{u}}^{n}$ in (1.14a), we have

$$
\begin{gathered}
\left\|\hat{\boldsymbol{u}}^{n}\right\|^{2}-\left\|\boldsymbol{u}^{n}\right\|^{2}+ \\
=2 \Delta t \int_{\Omega}\left(\nabla \cdot \hat{\boldsymbol{u}}^{n}-\boldsymbol{u}^{n}\left\|^{2}+2 \triangle t\right\| \nabla \phi^{n} d \boldsymbol{x} \equiv I,\right. \\
=2 \hat{\boldsymbol{u}}^{n} \|^{2}=-2 \triangle t \int_{\Omega} \hat{\boldsymbol{u}}^{n} \cdot \nabla \triangle \phi^{n} d \boldsymbol{x}
\end{gathered}
$$

Taking the divergence of the first equation in $(1.14 \mathrm{~b})$, we get

$$
\nabla \cdot \hat{\boldsymbol{u}}^{n}=\triangle\left(\phi^{n}-\phi^{n+1}\right) .
$$

Plugging back into the last term in the right hand side of (1.15), we have

$$
\begin{aligned}
I & =-2 \Delta t \int_{\Omega} \Delta\left(\phi^{n+1}-\phi^{n}\right) \Delta \phi^{n} d \boldsymbol{x} \\
& =-\Delta t\left(\left\|\Delta \phi^{n+1}\right\|^{2}-\left\|\Delta \phi^{n}\right\|^{2}\right)+\Delta t\left\|\Delta\left(\phi^{n+1}-\phi^{n}\right)\right\|^{2} \\
& =-\Delta t\left(\left\|\Delta \phi^{n+1}\right\|^{2}-\left\|\Delta \phi^{n}\right\|^{2}\right)+\Delta t\left\|\nabla \cdot \hat{\boldsymbol{u}}^{n}\right\|^{2},
\end{aligned}
$$

where in the last step we used (1.16) again. We note that $\left\|\nabla \cdot \hat{\boldsymbol{u}}^{n}\right\|$ can be controlled by the diffusion term $\left\|\nabla \hat{\boldsymbol{u}}^{n}\right\|$. The combination of (1.15) and (1.17) results in

$$
\left\|\hat{\boldsymbol{u}}^{n}\right\|^{2}-\left\|\boldsymbol{u}^{n}\right\|^{2}+\left\|\hat{\boldsymbol{u}}^{n}-\boldsymbol{u}^{n}\right\|^{2}+\triangle t\left\|\nabla \hat{\boldsymbol{u}}^{n}\right\|^{2}+\triangle t\left(\left\|\Delta \phi^{n+1}\right\|^{2}-\left\|\triangle \phi^{n}\right\|^{2}\right) \leq 0 .
$$

Next, we need an energy estimate of the first equation in (1.14b). As can be seen, the incompressibility of $\boldsymbol{u}^{n+1}$, together with the boundary condition $\boldsymbol{u}^{n+1} \cdot \boldsymbol{n}=0$ on $\partial \Omega$ for the normal component of $\boldsymbol{u}^{n+1}$, can guarantee that $u^{n+1}$ is orthogonal to the gradient of $\phi^{n}-\phi^{n+1}$, i.e.

$$
\int_{\Omega} \boldsymbol{u}^{n+1} \cdot \nabla\left(\phi^{n}-\phi^{n+1}\right) d \boldsymbol{x}=0 .
$$

If we take the inner product of the first equation in (1.14b) with $2 \boldsymbol{u}^{n+1}$, we have

$$
\left\|\boldsymbol{u}^{n+1}\right\|^{2}-\left\|\hat{\boldsymbol{u}}^{n}\right\|^{2}+\left\|\boldsymbol{u}^{n+1}-\hat{\boldsymbol{u}}^{n}\right\|^{2}=0 .
$$

Finally, the combination of (1.18) and (1.20) results in

$$
\left\|\boldsymbol{u}^{n+1}\right\|^{2}-\left\|\boldsymbol{u}^{n}\right\|^{2}+\triangle t\left\|\nabla \hat{\boldsymbol{u}}^{n}\right\|^{2}+\triangle t\left(\left\|\triangle \phi^{n+1}\right\|^{2}-\left\|\triangle \phi^{n}\right\|^{2}\right) \leq 0 .
$$

Then the proof is completed, to wit, the gauge method with explicit boundary conditions (1.10) is unconditionally stable for Stokes equations. The analysis in this paper follows the philosophy used above. 
Remark 1.1. The above arguments can also be applied in regard to the gauge method using the Dirichlet formulation. The only difference is that the boundary condition for the gauge variable analogous to (1.14b) will be $\phi^{n}-\phi^{n+1}=$ $\boldsymbol{\tau} \cdot \boldsymbol{u}^{n+1}=0$. Since $\boldsymbol{u}^{n+1}$ is divergence-free, (1.19) is still valid, which in turn yields (1.20). Equations (1.15)-(1.18) are the same. Finally, (1.21) still holds. In other words, the gauge method with explicit boundary conditions (1.10), in either the Neumann or Dirichlet formulation, is unconditionally stable for Stokes equations.

1.2. Connection between projection method and gauge method. The gauge method shares many similarities with the projection method [6]. The projection method has been thoroughly analyzed in [17, 18, 8, 22]. We will adopt analyses and techniques similar to those used in [8. One of the main differences between the gauge method and the projection method is that the gauge method is a direct discretization of the partial differential equations (1.5), while the projection method is a fractional splitting of the Navier-Stokes equations with some artificial numerical boundary conditions. Consequently, the projection method results in a singular perturbation of the original PDE and numerical boundary layers [14, 8. This subtle fact is reflected in our analysis of the numerical method by the fact that the consistency analysis of the gauge method is much easier than that of the projection method, with regular expansions of the numerical scheme, and no numerical boundary layers are included. Another advantage of the gauge method is that it overcomes some difficulties in the numerical computations of the incompressible flow, such as the approximate projection in the projection methods [1] and the pressure boundary conditions [10. Extension of the gauge method to the $3 \mathrm{D}$ case is also straightforward. Although we concentrate on the $2 \mathrm{D}$ case here for simplicity, any convergence analysis in this paper can easily be extended to the 3D gauge method.

This paper is organized as follows: Section 2 describes time and space discretizations using gauge formulation, Section 3 provides error analysis and estimates for the spatially continuous Stokes equations, Section 4 proves the convergence theorem for the full NSE in the spatially discrete case, and Section 5 comments on the Dirichlet formulation.

\section{Time AND SPACE DisCRETIZATIONS}

We will use the backward Euler method as our first order time discretization, the Crank-Nicolson method as our second order time discretization, and MAC grids as our spatial discretization. Since our analysis is close to that of the projection method, we adopt notation similar to that in [8].

\subsection{Time discretization.}

Backward Euler. The backward Euler time discretization of (1.8) with explicit boundary conditions for $\boldsymbol{a}$ can be written as

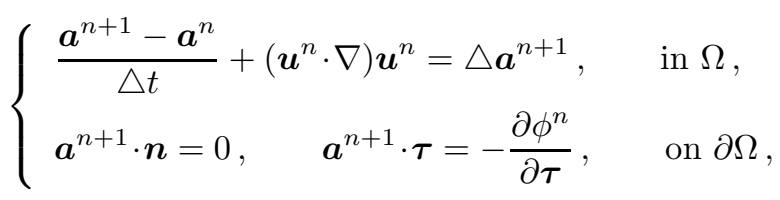


and

$$
\left\{\begin{array}{l}
\triangle \phi^{n+1}=-\nabla \cdot \boldsymbol{a}^{n+1}, \quad \text { in } \Omega, \\
\frac{\partial \phi^{n+1}}{\partial \boldsymbol{n}}=0, \quad \text { on } \partial \Omega,
\end{array}\right.
$$

and the velocity is given by

$$
\boldsymbol{u}^{n+1}=\boldsymbol{a}^{n+1}+\nabla \phi^{n+1} .
$$

Crank-Nicolson. We can also discretize (1.8) using second order Crank-Nicolson method, with explicit boundary conditions for $\boldsymbol{a}$,

$$
\begin{cases}\frac{\boldsymbol{a}^{n+1}-\boldsymbol{a}^{n}}{\triangle t}+\left(\boldsymbol{u}^{n+\frac{1}{2}} \cdot \nabla\right) \boldsymbol{u}^{n+\frac{1}{2}}=\triangle \frac{1}{2}\left(\boldsymbol{a}^{n}+\boldsymbol{a}^{n+1}\right), & \text { in } \Omega, \\ \boldsymbol{a}^{n+1} \cdot \boldsymbol{n}=0, \quad \boldsymbol{a}^{n+1} \cdot \boldsymbol{\tau}=-\left(2 \frac{\partial \phi^{n}}{\partial \boldsymbol{\tau}}-\frac{\partial \phi^{n-1}}{\partial \boldsymbol{\tau}}\right), & \text { on } \partial \Omega,\end{cases}
$$

where the term $\left(\boldsymbol{u}^{n+\frac{1}{2}} \cdot \nabla\right) \boldsymbol{u}^{n+\frac{1}{2}}$ is defined as $\frac{3}{2}\left(\boldsymbol{u}^{n} \cdot \nabla\right) \boldsymbol{u}^{n}-\frac{1}{2}\left(\boldsymbol{u}^{n-1} \cdot \nabla\right) \boldsymbol{u}^{n-1}$. On the boundary, $\boldsymbol{a}$ is determined by the second order one-sided extrapolation of $\phi$ in the previous time steps. $\phi^{n+1}$ at time $t^{n+1}$ is still determined by $\boldsymbol{a}$ via (2.2), and the velocity can be calculated by $(2.3)$.

Remark 2.1. As can be seen, if the implicit boundary condition for the auxiliary field $\boldsymbol{a}$ in the momentum equation is adopted - for example, if the implicit boundary conditions for $\boldsymbol{a}$ is imposed when we solve $\boldsymbol{a}$ by backward Euler time-discretization

$$
\begin{cases}\frac{\boldsymbol{a}^{n+1}-\boldsymbol{a}^{n}}{\triangle t}+\left(\boldsymbol{u}^{n} \cdot \nabla\right) \boldsymbol{u}^{n}=\triangle \boldsymbol{a}^{n+1}, & \text { in } \Omega, \\ \boldsymbol{a}^{n+1} \cdot \boldsymbol{n}=0, \quad \boldsymbol{a}^{n+1} \cdot \boldsymbol{\tau}=-\frac{\partial \phi^{n+1}}{\partial \boldsymbol{\tau}}, & \text { on } \partial \Omega,\end{cases}
$$

coupled with the kinematic equation

$$
\left\{\begin{array}{c}
\triangle \phi^{n+1}=-\nabla \cdot \boldsymbol{a}^{n+1}, \quad \text { in } \Omega, \\
\frac{\partial \phi^{n+1}}{\partial \boldsymbol{n}}=0, \quad \text { on } \partial \Omega, \\
\boldsymbol{u}^{n+1}=\boldsymbol{a}^{n+1}+\nabla \phi^{n+1}
\end{array}\right.
$$

- then by (2.3), the relation among the velocity $\boldsymbol{u}$, the auxiliary field $\boldsymbol{a}$ and the gauge variable $\phi,(2.5)-(2.7)$ can be rewritten as

$$
\left\{\begin{array}{l}
\frac{\boldsymbol{u}^{n+1}-\boldsymbol{u}^{n}}{\triangle t}+\left(\boldsymbol{u}^{n} \cdot \nabla\right) \boldsymbol{u}^{n}+\nabla \tilde{p}^{n+1}=\Delta \boldsymbol{u}^{n+1}, \quad \text { in } \Omega \\
\nabla \cdot \boldsymbol{u}^{n+1}=0, \quad \text { in } \Omega, \\
\boldsymbol{u}^{n+1}=0, \quad \text { on } \partial \Omega,
\end{array}\right.
$$

where

$$
\tilde{p}^{n+1}=-\frac{\phi^{n+1}-\phi^{n}}{\triangle t}+\triangle \phi^{n+1},
$$

which becomes the standard backward Euler discretization of the Navier-Stokes equations. The convergence of this scheme is straightforward. However, to implement the implicit boundary conditions in (2.5), one has to iterate the system 
between (2.5) and (2.6), which is very costly. Extensive computational evidence shows that this iteration is not necessary, and accuracy is still maintained with the explicit boundary conditions for $\boldsymbol{a}$ in (2.1). Our analysis will give a theoretical insight into this.

Dirichlet formulation. If we prescribe the Dirichlet boundary condition (1.7) of $\phi$, the corresponding first order scheme analogous to (2.1)-(2.3) becomes

$$
\begin{aligned}
& \begin{cases}\frac{\boldsymbol{a}^{n+1}-\boldsymbol{a}^{n}}{\triangle t}+\left(\boldsymbol{u}^{n} \cdot \nabla\right) \boldsymbol{u}^{n}=\Delta \boldsymbol{a}^{n+1}, & \text { in } \Omega, \\
\boldsymbol{a}^{n+1} \cdot \boldsymbol{n}=-\frac{\partial \phi^{n}}{\partial \boldsymbol{n}}, \quad \boldsymbol{a}^{n+1} \cdot \boldsymbol{\tau}=0, & \text { on } \partial \Omega,\end{cases} \\
& \begin{cases}\triangle \phi^{n+1}=-\nabla \cdot \boldsymbol{a}^{n+1}, & \text { in } \Omega, \\
\phi^{n+1}=0, \quad \text { on } \partial \Omega, & \end{cases} \\
& \boldsymbol{u}^{n+1}=\boldsymbol{a}^{n+1}+\nabla \phi^{n+1} .
\end{aligned}
$$

It is only necessary to solve three Poisson-like equations with Dirichlet boundary conditions. This gives some advantage in the iterative methods for the linear system generated by the finite element method [7. Similarly, the corresponding second order method using the Crank-Nicolson time discretization becomes

$$
\left\{\begin{array}{lc}
\frac{\boldsymbol{a}^{n+1}-\boldsymbol{a}^{n}}{\triangle t}+\left(\boldsymbol{u}^{n+\frac{1}{2}} \cdot \nabla\right) \boldsymbol{u}^{n+\frac{1}{2}}=\triangle \frac{1}{2}\left(\boldsymbol{a}^{n+1}+\boldsymbol{a}^{n}\right), & \text { in } \Omega, \\
\boldsymbol{a}^{n+1} \cdot \boldsymbol{n}=-2 \frac{\partial \phi^{n}}{\partial \boldsymbol{n}}+\frac{\partial \phi^{n-1}}{\partial \boldsymbol{n}}, \quad \boldsymbol{a}^{n+1} \cdot \boldsymbol{\tau}=0, & \text { on } \partial \Omega
\end{array}\right.
$$

along with (2.11), which gives us $\phi^{n+1}$ at the time step $t^{n+1}$, and (2.12), which updates the velocity $\boldsymbol{u}^{n+1}$.

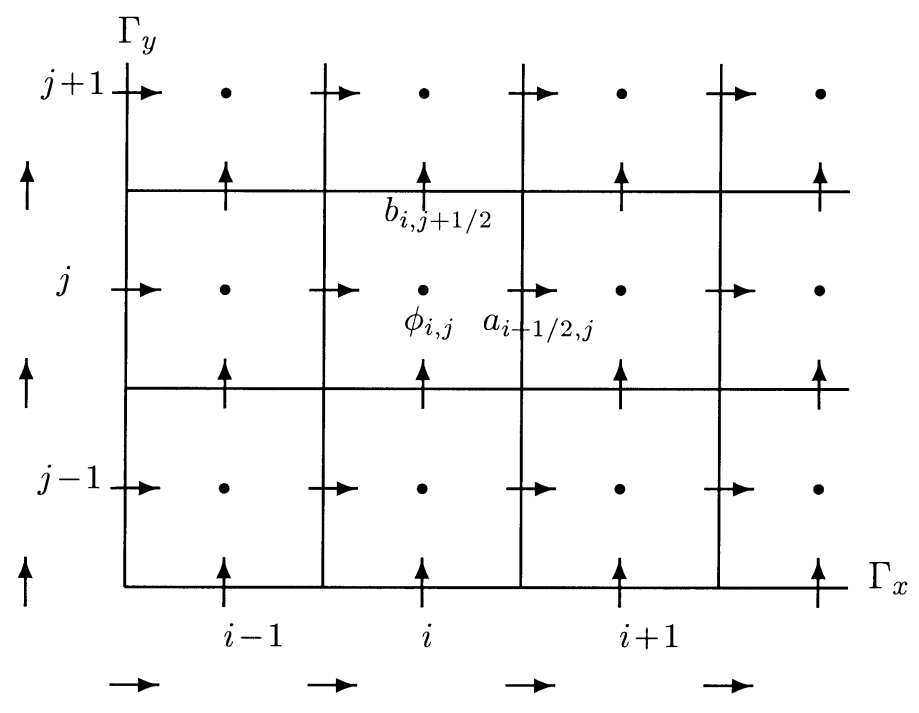

Figure 1. MAC mesh, Harlow, Welch, 1965 
We will show later that this Dirichlet gauge method with explicit boundary conditions is still stable. Yet, because of the lack of the normal compatibility on the boundary, there are some problems in the expansions of the numerical scheme. We can only get a $\sqrt{\triangle t}$ order error estimate. However, it is hoped that this is only a theoretical difficulty, which will not influence practical computations.

2.2. Space discretization. We will concentrate on the situation when $\Omega=$ $[-1,1] \times[0,2 \pi]$ with periodic boundary conditions in the $y$ direction and no-slip boundary conditions in the $x$ direction:

$$
\boldsymbol{u}(x, 0, t)=\boldsymbol{u}(x, 2 \pi, t), \quad \boldsymbol{u}(-1, y, t)=\boldsymbol{u}(1, y, t)=0 .
$$

$\partial^{\prime} \Omega$ is used to denote the part of the boundary at $x= \pm 1$. It is assumed that $\triangle x=\triangle y=h$. The analysis of the spatial discretization with standard grids is quite difficult. Some analysis of the projection method with standard grids was carried out by Wetton in [15]. In this paper, we only consider the MAC staggered grids for spatial discretization. An illustration of the MAC mesh near the boundary is given in Figure 1. Here the gauge variable $\phi$ (also the pressure $p$ ) is evaluated at the dot points $(i, j)$, the gauge variable $a$ (also the $u$ velocity) is evaluated at the right arrow points $(i \pm 1 / 2, j)$, and the gauge variable $b$ (also the $v$ velocity) is evaluated at the upper arrow points $(i, j \pm 1 / 2)$. The discrete divergence of $\boldsymbol{a}$ (also $\boldsymbol{u}$ and $\hat{\boldsymbol{u}})$ is computed at the dot points:

$$
\left(\nabla_{h} \cdot \boldsymbol{a}\right)_{i, j}=\frac{a_{i+1 / 2, j}-a_{i-1 / 2, j}}{h}+\frac{b_{i, j+1 / 2}-b_{i, j-1 / 2}}{h}
$$

Other differential operators are defined as follows (for brevity, we just write out the definition of these operators on $\boldsymbol{a}, \phi$, where the same definition can be applied to $\boldsymbol{u}, \hat{\boldsymbol{u}}$ and $p$ ):

$$
\begin{gathered}
\left(\triangle_{h} a\right)_{i+1 / 2, j}=\frac{a_{i+3 / 2, j}-2 a_{i+1 / 2, j}+a_{i-1 / 2, j}}{h^{2}} \\
+\frac{a_{i+1 / 2, j+1}-2 a_{i+1 / 2, j}+a_{i+1 / 2, j-1}}{h^{2}}, \\
\left(\triangle_{h} b\right)_{i, j+1 / 2}=\frac{b_{i+1, j+1 / 2}-2 b_{i, j+1 / 2}+b_{i-1, j+1 / 2}}{h^{2}} \\
+\frac{b_{i, j+3 / 2}-2 b_{i, j+1 / 2}+b_{i, j-1 / 2}}{h^{2}}, \\
\left(D_{x} \phi\right)_{i+1 / 2, j}=\frac{\phi_{i+1, j}-\phi_{i, j}, \quad\left(D_{y} \phi\right)_{i, j+1 / 2}=\frac{\phi_{i, j+1}-\phi_{i, j}}{h},}{h} \\
\bar{a}_{i, j+1 / 2}=\frac{1}{4}\left(a_{i+1 / 2, j}+a_{i-1 / 2, j}+a_{i+1 / 2, j+1}+a_{i-1 / 2, j+1}\right), \\
\bar{b}_{i+1 / 2, j}=\frac{1}{4}\left(b_{i+1, j+1 / 2}+b_{i+1, j-1 / 2}+b_{i, j+1 / 2}+b_{i, j-1 / 2}\right), \\
\mathcal{N}_{h}(\boldsymbol{u}, a)_{i+1 / 2, j}=u_{i+1 / 2, j} \frac{a_{i+3 / 2, j}-a_{i-1 / 2, j}}{2 h}+\bar{v}_{i+1 / 2, j} \frac{a_{i+1 / 2, j+1}-a_{i+1 / 2, j-1}}{2 h}
\end{gathered}
$$




$$
\mathcal{N}_{h}(\boldsymbol{u}, b)_{i, j+1 / 2}=\bar{u}_{i, j+1 / 2} \frac{b_{i+1, j+1 / 2}-b_{i-1, j+1 / 2}}{2 h}+v_{i, j+1 / 2} \frac{b_{i, j+3 / 2}-b_{i, j-1 / 2}}{2 h} .
$$

Clearly the truncation errors of these approximations are of second order. The first momentum equation (for $a$ ) is implemented at right arrow points, the second momentum equation is implemented at upper arrow points, and the (discrete) Poisson equation for $\phi$ is implemented at dot points.

The boundary condition $u=0$ is imposed at the vertical physical boundary $\Gamma_{y}$, whereas $v=0$ is imposed by $v_{0, j+1 / 2}+v_{1, j-1 / 2}=0$. Similarly, the boundary condition $v=0$ is imposed at the horizontal physical boundary $\Gamma_{y}$, where $u=0$ is imposed by $u_{i+1 / 2,0}+u_{i-1 / 2,1}=0$. Consequently, the boundary condition $a=$ $0, b=-\partial_{y} \phi$ at the left vertical boundary is implemented by

$$
a=0, \quad b_{1, j+1 / 2}+b_{0, j+1 / 2}=-\frac{\phi_{1, j+1}-\phi_{1, j}}{h}-\frac{\phi_{0, j+1}-\phi_{0, j}}{h} .
$$

Similar boundary conditions for $\boldsymbol{a}$ are imposed at the other three boundaries.

One of the main advantage of the MAC grids is that the spatial discretization of $(2.2)$ and (2.3),

$$
\triangle_{h} \phi^{n+1}=-\nabla_{h} \cdot \boldsymbol{a}^{n+1}, \quad \boldsymbol{u}^{n+1}=\boldsymbol{a}^{n+1}+\nabla_{h} \phi^{n+1},
$$

gives an exact projection

$$
\boldsymbol{a}^{n+1}=\boldsymbol{u}^{n+1}-\nabla_{h} \phi^{n+1}, \quad \nabla_{h} \cdot \boldsymbol{u}^{n+1}=0,
$$

and the Neumann boundary condition

$$
\frac{\partial \phi^{n+1}}{\partial \boldsymbol{n}}=0, \quad \text { on } \partial \Omega,
$$

gives the boundary condition for the normal component of $\boldsymbol{u}$,

$$
\boldsymbol{n} \cdot \boldsymbol{u}^{n+1}=0, \quad \text { on } \partial^{\prime} \Omega .
$$

Therefore we can rewrite the full discrete scheme analogous to (2.1)-(2.3) in the following form, which will be used in the convergence and error analysis:

$$
\begin{gathered}
\left\{\begin{array}{l}
\frac{\boldsymbol{a}^{n+1}-\boldsymbol{a}^{n}}{\Delta t}+\mathcal{N}_{h}\left(\boldsymbol{u}^{n}, \boldsymbol{u}^{n}\right)=\Delta_{h} \boldsymbol{a}^{n+1}, \quad \text { in } \Omega, \\
\boldsymbol{a}^{n+1}=-\nabla_{h} \phi^{n}, \quad \text { on } \partial^{\prime} \Omega,
\end{array}\right. \\
\left\{\begin{array}{l}
\boldsymbol{u}^{n+1}=\boldsymbol{a}^{n+1}+\nabla_{h} \phi^{n+1}, \\
\nabla_{h} \cdot \boldsymbol{u}^{n+1}=0, \quad \text { in } \Omega, \\
\boldsymbol{n} \cdot \boldsymbol{u}^{n+1}=0,
\end{array} \quad \text { on } \partial^{\prime} \Omega .\right.
\end{gathered}
$$

\section{Spatially CONTinuous Case for stokes equations}

We have already shown the unconditional stability of the gauge method with explicit boundary conditions in the introduction. Now our convergence theorem for Stokes equations is stated. 
Theorem 3.1. Let $(\boldsymbol{u}, \phi)$ be a smooth solution of Stokes equations with smooth initial data $\boldsymbol{u}^{0}(\boldsymbol{x})$ and let $\left(\boldsymbol{u}_{\triangle t}, \phi_{\triangle t}\right)$ be the numerical solution of the semi-discrete gauge method with explicit boundary conditions (1.10)-(1.13). Then

$$
\left\|\boldsymbol{u}-\boldsymbol{u}_{\triangle t}\right\|_{L^{\infty}\left(0, T ; L^{2}\right)} \leq C \triangle t
$$

The convergence proof follows the standard strategy of consistency and stability estimates. We have already proven the stability of the scheme in the introduction. In the consistency part, we first make a transformation of the numerical scheme. Instead of directly comparing the numerical solutions with the exact solutions, we compare them with the ones constructed from the exact field, $\phi$. The constructed fields satisfy the boundary conditions in the numerical scheme exactly. The advantage of this approach is that no error term appears in the boundary conditions. This simplifies the energy estimates used in the stability part of the proof.

For simplicity, we just do first order expansions in the spatially continuous case. In the fully discrete case, second order expansions are required to establish the a priori estimates needed in the convergence proof.

3.1. Truncation error and consistency analysis of the numerical solutions. We follow the strategy of Strang [19] in constructing a high order expansion from the exact solutions to satisfy the numerical scheme up to high order. This will enable us to give a sharper a priori estimate.

By introducing the new variable $\hat{\boldsymbol{u}}^{n}=\boldsymbol{a}^{n+1}+\nabla \phi^{n}$, we obtained (1.14), an equivalent reformulation of the scheme (1.13), (1.11), (1.12).

Let $\boldsymbol{u}_{\mathrm{e}}(\boldsymbol{x}, t)$ and $p_{\mathrm{e}}(\boldsymbol{x}, t)$ be the exact solutions of the Stokes equations, i.e.

$$
\left\{\begin{array}{l}
\partial_{t} \boldsymbol{u}_{\mathrm{e}}+\nabla p_{\mathrm{e}}=\Delta \boldsymbol{u}_{\mathrm{e}}, \quad \text { in } \Omega, \\
\nabla \cdot \boldsymbol{u}_{\mathrm{e}}=0, \quad \text { in } \Omega, \\
\boldsymbol{u}_{\mathrm{e}}=0, \quad \text { on } \partial \Omega,
\end{array}\right.
$$

and let $\phi_{\mathrm{e}}(\boldsymbol{x}, t)$ be a solution of the following heat equation with Neumann boundary condition:

$$
\left\{\begin{array}{l}
\partial_{t} \phi_{\mathrm{e}}=\Delta \phi_{\mathrm{e}}-p_{\mathrm{e}}, \quad \text { in } \Omega \\
\frac{\partial \phi_{\mathrm{e}}}{\partial \boldsymbol{n}}=0, \quad \text { on } \partial \Omega
\end{array}\right.
$$

where the initial data $\phi_{\mathrm{e}}(\boldsymbol{x}, 0)$ is chosen from the following Poisson equation:

$$
\begin{cases}\triangle \phi_{\mathrm{e}}(\boldsymbol{x}, 0) & =p_{\mathrm{e}}(\boldsymbol{x}, 0)+C_{1}, \\ \frac{\partial \phi_{\mathrm{e}}(\boldsymbol{x}, 0)}{\partial \boldsymbol{n}}=0, & \text { on } \partial \Omega,\end{cases}
$$

where $C_{1}$ is a constant such that $C_{1}=-\int_{\Omega} p_{\mathrm{e}}(\boldsymbol{x}, 0) d \boldsymbol{x}$, to maintain the consistency that follows from the Neumann boundary condition. Obviously, if we introduce $\boldsymbol{a}_{\mathrm{e}}=\boldsymbol{u}_{\mathrm{e}}-\nabla \phi_{\mathrm{e}}$, then $\left(\boldsymbol{a}_{\mathrm{e}}, \phi_{\mathrm{e}}\right)$ is an exact solution of the Stokes equations in gauge formulation. 
Next, we let $\boldsymbol{u}_{1}$ be a solution of the Stokes equations with the prescribed boundary conditions and initial data

$$
\left\{\begin{array}{l}
\partial_{t} \boldsymbol{u}_{1}+\nabla p_{1}=\triangle \boldsymbol{u}_{1}, \quad \text { in } \Omega, \\
\nabla \cdot \boldsymbol{u}_{1}=0, \quad \text { in } \Omega, \\
\boldsymbol{u}_{1}=\partial_{t} \nabla \phi_{\mathrm{e}}, \quad \text { on } \partial \Omega, \\
\boldsymbol{u}_{1}(\boldsymbol{x}, 0)=0 .
\end{array}\right.
$$

By the construction of $\phi_{\mathrm{e}}(\boldsymbol{x}, 0)$, we have

$$
\partial_{t} \phi_{\mathrm{e}}(\boldsymbol{x}, 0)=\triangle \phi_{\mathrm{e}}(\boldsymbol{x}, 0)-p_{\mathrm{e}}(\boldsymbol{x}, 0)=C_{1}, \quad \text { on } \partial \Omega,
$$

which implies that $\partial_{t} \nabla \phi_{\mathrm{e}}(\boldsymbol{x}, 0)=0$ on the boundary, so we can choose $\boldsymbol{u}_{1}(\boldsymbol{x}, 0)=0$ as in (3.5).

Consequently, we let

$$
\hat{\boldsymbol{u}}_{1}=\boldsymbol{u}_{1}+\partial_{t} \boldsymbol{a}_{\mathrm{e}}
$$

and construct approximate profiles

$$
U^{*}=\boldsymbol{u}_{\mathrm{e}}+\triangle t \hat{\boldsymbol{u}}_{1}, \quad U=\boldsymbol{u}_{\mathrm{e}}+\Delta t \boldsymbol{u}_{1}, \quad \Phi=\phi_{\mathrm{e}} .
$$

Lemma 3.1. We have

$$
\begin{aligned}
& \left\{\begin{array}{l}
\frac{U^{* n}-U^{n}}{\Delta t}+\Delta \nabla \Phi^{n}=\Delta U^{* n}+\Delta t \boldsymbol{f}^{n}, \quad \text { in } \Omega, \\
U^{* n}=0, \quad \text { on } \partial \Omega,
\end{array}\right. \\
& \left\{\begin{array}{l}
U^{n+1}-U^{* n}+\nabla\left(\Phi^{n}-\Phi^{n+1}\right)=\Delta t^{2} \boldsymbol{g}^{n}, \quad \text { in } \Omega, \\
\nabla \cdot U^{n+1}=0, \quad \text { in } \Omega, \\
\frac{\partial\left(\Phi^{n}-\Phi^{n+1}\right)}{\partial \boldsymbol{n}}=\boldsymbol{n} \cdot U^{n+1}=0, \quad \text { on } \partial \Omega,
\end{array}\right. \\
& U^{0}=\boldsymbol{u}^{0}, \quad \text { in } \Omega,
\end{aligned}
$$

where $\boldsymbol{f}^{n}$ and $\boldsymbol{g}^{n}$ are bounded functions.

Proof. Substituting (3.8) into (3.9a), by direct calculations we obtain

$$
\begin{aligned}
\frac{U^{* n}-U^{n}}{\triangle t}+\triangle \nabla \Phi^{n}-\triangle U^{* n} & =\hat{\boldsymbol{u}}_{1}-\boldsymbol{u}_{1}+\triangle \nabla \phi_{\mathrm{e}}-\triangle \boldsymbol{u}_{\mathrm{e}}-\triangle t \triangle \hat{\boldsymbol{u}}_{1} \\
& =\left(\partial_{t} \boldsymbol{a}_{\mathrm{e}}-\triangle \boldsymbol{a}_{\mathrm{e}}\right)-\triangle t \triangle \hat{\boldsymbol{u}}_{1} \\
& =-\triangle t \triangle \hat{\boldsymbol{u}}_{1}=O(\triangle t), \quad \text { in } \Omega .
\end{aligned}
$$

In the last step we used the fact that $\left(\boldsymbol{u}_{\mathrm{e}}, \boldsymbol{a}_{\mathrm{e}}\right)$ is the exact solution of the Stokes equations in gauge formulation, i.e.

$$
\partial_{t} \boldsymbol{a}_{\mathrm{e}}-\triangle \boldsymbol{a}_{\mathrm{e}}=0, \quad \text { in } \Omega .
$$

By the construction of $\hat{\boldsymbol{u}}_{1}$ and the boundary condition for $\boldsymbol{u}_{1}$, we have

$$
\hat{\boldsymbol{u}}_{1}^{n}=\boldsymbol{u}_{1}^{n}+\partial_{t} \boldsymbol{a}_{\mathrm{e}}^{n}=\partial_{t} \boldsymbol{u}_{\mathrm{e}}^{n}=0, \quad \text { on } \partial \Omega,
$$

which shows that

$$
U^{* n}=0, \quad \text { on } \partial \Omega .
$$


For the equation in (3.9b), by direct calculations and Taylor expansions of $U$ and $\Phi$ w.r.t. time $t$, we have

$$
\begin{aligned}
& U^{n+1}-U^{* n}+\nabla\left(\Phi^{n}-\Phi^{n+1}\right) \\
& =\boldsymbol{u}_{\mathrm{e}}^{n}+\triangle t \partial_{t} \boldsymbol{u}_{\mathrm{e}}^{n}+\triangle t \boldsymbol{u}_{1}^{n}+O\left(\triangle t^{2}\right)-\boldsymbol{u}_{\mathrm{e}}^{n}-\triangle t \hat{\boldsymbol{u}}_{1}^{n}-\triangle t \partial_{t} \nabla \phi_{\mathrm{e}}^{n}+O\left(\triangle t^{2}\right) \\
& =\triangle t \partial_{t} \boldsymbol{a}_{\mathrm{e}}^{n}+\triangle t\left(\boldsymbol{u}_{1}^{n}-\hat{\boldsymbol{u}}_{1}^{n}\right)+O\left(\triangle t^{2}\right) \\
& =O\left(\triangle t^{2}\right), \quad \text { in } \Omega .
\end{aligned}
$$

Since both $\boldsymbol{u}_{\mathrm{e}}$ and $\boldsymbol{u}_{1}$ are divergence free, we obtain

$$
\nabla \cdot U^{n+1}=0, \quad \text { in } \Omega,
$$

and by the construction of our $U$ and $\Phi$, we have

$$
\frac{\partial \Phi^{n+1}}{\partial \boldsymbol{n}}=\boldsymbol{n} \cdot U^{n+1}=0, \quad \text { on } \partial \Omega .
$$

Then we complete the consistency analysis of the first order gauge method with explicit boundary conditions. Lemma 3.1 is proven.

3.2. Proof of Theorem 3.1. We define the error functions

$$
\boldsymbol{e}^{n}=U^{n}-\boldsymbol{u}^{n}, \quad \hat{\boldsymbol{e}}^{n}=U^{* n}-\hat{\boldsymbol{u}}^{n}, \quad q^{n}=\Phi^{n}-\phi^{n} .
$$

In Section 1, by making a transformation, we got (1.14), which is an equivalent formulation of (1.13), (1.11), (1.12). Subtracting (3.9) from (1.14), we get the equations for the error functions:

$$
\begin{aligned}
& \left\{\begin{array}{l}
\frac{\hat{\boldsymbol{e}}^{n}-\boldsymbol{e}^{n}}{\Delta t}=\Delta \hat{\boldsymbol{e}}^{n}-\nabla \Delta q^{n}+\Delta t \boldsymbol{f}^{n}, \quad \text { in } \Omega, \\
\hat{\boldsymbol{e}}^{n}=0, \quad \text { on } \partial \Omega,
\end{array}\right. \\
& \left\{\begin{array}{l}
\boldsymbol{e}^{n+1}-\hat{\boldsymbol{e}}^{n}+\nabla\left(q^{n}-q^{n+1}\right)=\Delta t^{2} \boldsymbol{g}^{n}, \quad \text { in } \Omega, \\
\nabla \cdot \boldsymbol{e}^{n+1}=0, \quad \text { in } \Omega, \\
\frac{\partial\left(q^{n}-q^{n+1}\right)}{\partial \boldsymbol{n}}=\boldsymbol{e}^{n+1} \cdot \boldsymbol{n}=0, \quad \text { on } \partial \Omega, \\
\boldsymbol{e}^{0}=0, \quad \text { in } \Omega .
\end{array}\right.
\end{aligned}
$$

It can be seen that the system (3.18) is very similar to (1.14), except for the local truncation error terms $\Delta t \boldsymbol{f}^{n}, \Delta t^{2} \boldsymbol{g}^{n}$. So most of the energy estimate techniques we used in Section 1 can be carried out here similarly. The estimates corresponding to the local error terms can be given by the Cauchy inequality. We will omit some of the details in the following analysis.

Taking the inner product of (3.18a) with $2 \hat{\boldsymbol{e}}^{n}$ and using the fact that $\hat{\boldsymbol{e}}^{n}$ vanishes on the boundary, we have

$$
\begin{aligned}
\left\|\hat{\boldsymbol{e}}^{n}\right\|^{2}-\left\|\boldsymbol{e}^{n}\right\|^{2}+\left\|\hat{\boldsymbol{e}}^{n}-\boldsymbol{e}^{n}\right\|^{2}+2 \Delta t\left\|\nabla \hat{\boldsymbol{e}}^{n}\right\|^{2} \\
\quad \leq \Delta t^{3}\left\|\boldsymbol{f}^{n}\right\|^{2}+\triangle t\left\|\hat{\boldsymbol{e}}^{n}\right\|^{2}-2 \triangle t \int_{\Omega} \hat{\boldsymbol{e}}^{n} \cdot \nabla \Delta q^{n} d \boldsymbol{x} .
\end{aligned}
$$


Taking the inner product of the first equation in (3.18b) with $2 e^{n+1}$ and using a similar argument as in Section 1, i.e., that $\boldsymbol{e}^{n+1}$ is orthogonal to the gradient of $q^{n}-q^{n+1}$, we arrive at

$$
\left\|\boldsymbol{e}^{n+1}\right\|^{2}-\left\|\hat{\boldsymbol{e}}^{n}\right\|^{2}+\left\|\boldsymbol{e}^{n+1}-\hat{\boldsymbol{e}}^{n}\right\|^{2} \leq \triangle t\left\|\boldsymbol{e}^{n+1}\right\|^{2}+\triangle t^{3}\left\|\boldsymbol{g}^{n}\right\|^{2} .
$$

Combining (3.19) and (3.20), we get

$$
\begin{aligned}
& \left\|\boldsymbol{e}^{n+1}\right\|^{2}-\left\|\boldsymbol{e}^{n}\right\|^{2}+\left\|\hat{\boldsymbol{e}}^{n}-\boldsymbol{e}^{n}\right\|^{2}+\left\|\boldsymbol{e}^{n+1}-\hat{\boldsymbol{e}}^{n}\right\|^{2}+2 \triangle t\left\|\nabla \hat{\boldsymbol{e}}^{n}\right\|^{2} \\
& \leq C \triangle t\left(\left\|\boldsymbol{e}^{n}\right\|^{2}+\left\|\boldsymbol{e}^{n+1}\right\|^{2}\right)+\Delta t^{3}\left(\left\|\boldsymbol{f}^{n}\right\|^{2}+\left\|\boldsymbol{g}^{n}\right\|^{2}\right) \\
& \quad-2 \triangle t \int_{\Omega} \hat{\boldsymbol{e}}^{n} \cdot \nabla \Delta q^{n} d \boldsymbol{x} .
\end{aligned}
$$

Similarly to the analysis in (1.17), the estimate of the last term in (3.21) is determined by integration by parts and then using the first equation in (3.18b):

$$
\begin{aligned}
I \equiv & -2 \Delta t \int_{\Omega} \hat{\boldsymbol{e}}^{n} \cdot \nabla \Delta q^{n} d \boldsymbol{x} \\
= & 2 \Delta t \int_{\Omega}\left(\nabla \cdot \hat{\boldsymbol{e}}^{n}\right) \Delta q^{n} d \boldsymbol{x} \\
= & -2 \triangle t \int_{\Omega} \Delta\left(q^{n+1}-q^{n}\right) \Delta q^{n} d \boldsymbol{x}-2 \triangle t^{3} \int_{\Omega}\left(\nabla \cdot \boldsymbol{g}^{n}\right) \triangle q^{n} d \boldsymbol{x} \\
= & -\Delta t\left(\left\|\Delta q^{n+1}\right\|^{2}-\left\|\Delta q^{n}\right\|^{2}\right)+\triangle t\left\|\Delta\left(q^{n+1}-q^{n}\right)\right\|^{2} \\
& -2 \triangle t^{3} \int_{\Omega}\left(\nabla \cdot \boldsymbol{g}^{n}\right) \triangle q^{n} d \boldsymbol{x} \\
= & -\triangle t\left(\left\|\Delta q^{n+1}\right\|^{2}-\left\|\Delta q^{n}\right\|^{2}\right)+\triangle t\left\|\nabla \cdot \hat{\boldsymbol{e}}^{n}\right\|^{2}+\Delta t^{5}\left\|\boldsymbol{g}^{n}\right\|^{2} \\
& +2 \triangle t^{3} \int_{\Omega}\left(\nabla \cdot \hat{\boldsymbol{e}}^{n}\right)\left(\nabla \cdot \boldsymbol{g}^{n}\right) d \boldsymbol{x}-2 \triangle t^{3} \int_{\Omega}\left(\nabla \cdot \boldsymbol{g}^{n}\right) \triangle q^{n} d \boldsymbol{x},
\end{aligned}
$$

By (3.22),

$$
\begin{aligned}
I \leq & -\Delta t\left(\left\|\Delta q^{n+1}\right\|^{2}-\left\|\Delta q^{n}\right\|^{2}\right)+\triangle t\left\|\nabla \hat{\boldsymbol{e}}^{n}\right\|^{2}+\triangle t^{2}\left\|\Delta q^{n}\right\|^{2} \\
& +2 \triangle t^{4}\left\|\nabla \cdot \boldsymbol{g}^{n}\right\|^{2}+\Delta t^{2}\left\|\nabla \hat{\boldsymbol{e}}^{n}\right\|^{2}+\triangle t^{5}\left\|\boldsymbol{g}^{n}\right\|^{2} .
\end{aligned}
$$

Going back to (3.21), we obtain

$$
\begin{aligned}
\left\|\boldsymbol{e}^{n+1}\right\|^{2}-\left\|\boldsymbol{e}^{n}\right\|^{2}+\triangle t\left\|\nabla \hat{\boldsymbol{e}}^{n}\right\|^{2}+\triangle t\left(\left\|\triangle q^{n+1}\right\|^{2}-\left\|\triangle q^{n}\right\|^{2}\right) \\
\leq C \triangle t\left(\left\|\boldsymbol{e}^{n}\right\|^{2}+\left\|\boldsymbol{e}^{n+1}\right\|^{2}\right)+\triangle t^{2}\left\|\triangle q^{n}\right\|^{2} \\
+C \triangle t^{3}\left(\left\|\boldsymbol{f}^{n}\right\|^{2}+\left\|\boldsymbol{g}^{n}\right\|^{2}+\triangle t\left\|\boldsymbol{g}^{n}\right\|_{H^{1}}^{2}\right) .
\end{aligned}
$$

Applying the discrete Grownwall lemma to the last inequality, we arrive at

$$
\left\|\boldsymbol{e}^{n}\right\|+\triangle t^{1 / 2}\left\|\nabla \hat{\boldsymbol{e}}^{n}\right\|+\triangle t^{1 / 2}\left\|\triangle q^{n}\right\| \leq C \triangle t,
$$

which completes the proof of Theorem 3.1.

\section{Spatially discrete Case for the full Navier-Stokes equations}

Theorem 4.1. Let $(\boldsymbol{u}, \phi)$ be a smooth solution of the Navier-Stokes equations (1.1) with smooth initial data $\boldsymbol{u}^{0}(\boldsymbol{x})$, and let $\left(\boldsymbol{u}_{h}, \phi_{h}\right)$ be the numerical solution of the gauge method (2.15) coupled with the MAC spatial discretizations. Assume the CFL constraint $\triangle t \leq C h$ for some suitable constant $C$ which we will specify in detail later. Then we have

$$
\left\|\boldsymbol{u}-\boldsymbol{u}_{h}\right\|_{L^{\infty}} \leq C\left(\triangle t+h^{2}\right)
$$


Some notation. For $\boldsymbol{a}=(a, b), \boldsymbol{c}=(c, d), \boldsymbol{u}=(u, v)$, we define the following discrete inner products on the MAC grids:

$$
\begin{aligned}
& \langle\boldsymbol{a}, \boldsymbol{c}\rangle=h^{2} \sum_{i=1}^{N-1} \sum_{j=1}^{N} a_{i+1 / 2, j} c_{i+1 / 2, j}+h^{2} \sum_{i=1}^{N} \sum_{j=1}^{N} b_{i, j+1 / 2} d_{i, j+1 / 2}, \\
& \left\langle\boldsymbol{u}, \nabla_{h} \phi\right\rangle=h \sum_{i=1}^{N-1} \sum_{j=1}^{N} u_{i+1 / 2, j}\left(\phi_{i+1, j}-\phi_{i, j}\right)+h \sum_{i=1}^{N} \sum_{j=1}^{N} v_{i, j+1 / 2}\left(\phi_{i, j+1}-\phi_{i, j}\right), \\
& \left\langle\nabla_{h} \cdot \boldsymbol{u}, \phi\right\rangle=h \sum_{i=1}^{N-1} \sum_{j=1}^{N}\left(u_{i+1 / 2, j}-u_{i-1 / 2, j}\right) \phi_{i, j}+h \sum_{i=1}^{N} \sum_{j=1}^{N}\left(v_{i, j+1 / 2}-v_{i, j-1 / 2}\right) \phi_{i, j},
\end{aligned}
$$

and discrete norms

$$
\|\boldsymbol{u}\|=\langle\boldsymbol{u}, \boldsymbol{u}\rangle^{1 / 2}, \quad\|\boldsymbol{u}\|_{\infty}=\max _{i, j}\left|\boldsymbol{u}_{i, j}\right| .
$$

Next we state some preliminary lemmas excerpted from [8] which are needed in the proof of the theorem.

Lemma 4.1. We have the following:

(i) Inverse inequality:

$$
\|f\|_{\infty} \leq \frac{C}{h}\|f\|
$$

(ii) Poincaré inequality: If $\left.f\right|_{x= \pm 1}=0$, then

$$
\|f\| \leq C\left\|\nabla_{h} f\right\| .
$$

(iii) If $\left.\boldsymbol{n} \cdot \boldsymbol{u}\right|_{x= \pm 1}=0$, then

$$
\left\langle\boldsymbol{u}, \nabla_{h} \phi\right\rangle=-\left\langle\nabla_{h} \cdot \boldsymbol{u}, \phi\right\rangle .
$$

(iv) If $\left.\boldsymbol{u}\right|_{x= \pm 1}=0$, then

$$
2\left\langle\boldsymbol{u}, \Delta_{h} \boldsymbol{u}\right\rangle \leq-\left\|\nabla_{h} \boldsymbol{u}\right\|^{2}-\left\|\nabla_{h} \cdot \boldsymbol{u}\right\|^{2} .
$$

(v) If $\left.\boldsymbol{a}\right|_{x= \pm 1}=0$ and $\left.\boldsymbol{c} \cdot \boldsymbol{n}\right|_{\boldsymbol{x}= \pm 1}=0$, then

$$
\left|\left\langle\boldsymbol{a}, \mathcal{N}_{h}(\boldsymbol{u}, \boldsymbol{c})\right\rangle\right| \leq C\|\boldsymbol{c}\|\left\|\nabla_{h} \boldsymbol{a}\right\|\|\boldsymbol{u}\|_{W^{1, \infty}} .
$$

Lemma 4.2. Let $(\boldsymbol{u}, p)$ be a solution of the Navier-Stokes equations with smooth initial data $\boldsymbol{u}^{0}(\boldsymbol{x})$. Let $\left(\boldsymbol{u}_{0}, p_{0}\right)$ be a solution of the following system:

$$
\left\{\begin{array}{l}
\partial_{t} \boldsymbol{u}_{0}+\nabla_{h} p_{0}+\mathcal{N}_{h}\left(\boldsymbol{u}_{0}, \boldsymbol{u}_{0}\right)=\Delta_{h} \boldsymbol{u}_{0}, \quad \text { in } \Omega \\
\nabla_{h} \cdot \boldsymbol{u}_{0}=0, \quad \text { in } \Omega \\
\boldsymbol{u}_{0}=0, \quad \text { at } \quad x= \pm 1 \\
\boldsymbol{u}_{0}(\cdot, 0)=\boldsymbol{u}^{0}(\cdot), \quad \text { in } \Omega
\end{array}\right.
$$

Then $\left(\boldsymbol{u}_{0}, \phi_{0}\right)$ is smooth in the sense that its discrete derivatives are bounded. Moreover,

$$
\left\|\boldsymbol{u}-\boldsymbol{u}_{0}\right\|_{L^{\infty}}+\left\|p-p_{0}\right\|_{L^{\infty}} \leq C h^{2} .
$$


Remark 4.1. Let $\phi_{0}$ be the solution of the following discrete heat equation:

$$
\left\{\begin{array}{l}
\partial_{t} \phi_{0}-\Delta_{h} \phi_{0}+p_{0}=0, \quad \text { in } \Omega, \\
\frac{\partial \phi_{0}}{\partial \boldsymbol{x}}=0, \quad \text { at } \quad x= \pm 1, \\
\phi_{0}(\cdot, 0)=\phi^{0}(\cdot), \quad \text { in } \Omega,
\end{array}\right.
$$

and define

$$
\boldsymbol{a}^{0}=\boldsymbol{u}^{0}-\nabla_{h} \phi^{0} .
$$

Then the solution $\left(\boldsymbol{u}_{0}, \phi_{0}\right)$ of the decoupled system (4.9), (4.11) is smooth in the sense that its discrete derivatives are bounded and

$$
\left\|\boldsymbol{u}-\boldsymbol{u}_{0}\right\|_{L^{\infty}}+\left\|\phi-\phi_{0}\right\|_{L^{\infty}} \leq C h^{2},
$$

where $(\boldsymbol{u}, \phi)$ is the solution of Navier-Stokes equations in the gauge formulation with initial data $\boldsymbol{u}^{0}$.

Lemma 4.3. Let $(\boldsymbol{u}, p)$ be a solution of the linear system of $O D E$

$$
\left\{\begin{array}{l}
\partial_{t} \boldsymbol{u}+\nabla_{h} p+\mathcal{N}_{h}\left(\boldsymbol{u}_{0}, \boldsymbol{u}\right)+\mathcal{N}_{h}\left(\boldsymbol{u}, \boldsymbol{u}_{0}\right)=\Delta_{h} \boldsymbol{u}+\boldsymbol{f}, \quad \text { in } \Omega \\
\nabla_{h} \cdot \boldsymbol{u}=0, \quad \text { in } \Omega \\
\boldsymbol{u}=\boldsymbol{g}, \quad \text { at } x= \pm 1 \\
\boldsymbol{u}(\cdot, 0)=\boldsymbol{u}^{0}(\cdot), \quad \text { in } \Omega
\end{array}\right.
$$

where $\boldsymbol{f}, \boldsymbol{g}$, and $\boldsymbol{u}_{0}$ are smooth and satisfy some compatibility conditions. Then $(\boldsymbol{u}, p)$ is smooth in the sense that its divided differences of various orders are bounded.

Remark 4.2. Once again, let $\phi$ be the solution of the discrete heat equation

$$
\left\{\begin{array}{l}
\partial_{t} \phi-\Delta_{h} \phi+p=0, \quad \text { in } \Omega, \\
\frac{\partial \phi}{\partial \boldsymbol{n}}=0, \quad \text { on } \partial^{\prime} \Omega .
\end{array}\right.
$$

Then the solution $(\boldsymbol{u}, \phi)$ of the decoupled system (4.14), (4.15) is also smooth in the sense that its divided differences of various orders are bounded.

4.1. Consistency analysis of spatial discretization with MAC grid. As pointed out in Section 2, the numerical scheme can be written in the form (2.15) for the convenience of our analysis. Similarly to the spatially continuous case, if we introduce $\hat{\boldsymbol{u}}^{n}=\boldsymbol{a}^{n+1}+\nabla_{h} \phi^{n},(2.15)$ is equivalent to

$$
\begin{aligned}
& \left\{\begin{array}{l}
\frac{\hat{\boldsymbol{u}}^{n}-\boldsymbol{u}^{n}}{\triangle t}+\mathcal{N}_{h}\left(\boldsymbol{u}^{n}, \boldsymbol{u}^{n}\right)+\triangle_{h} \nabla_{h} \phi^{n}=\triangle_{h} \hat{\boldsymbol{u}}^{n}, \quad \text { in } \Omega, \\
\hat{\boldsymbol{u}}^{n}=0, \quad \text { at } x= \pm 1, \quad \text { in } \Omega,
\end{array}\right. \\
& \left\{\begin{array}{l}
\boldsymbol{u}^{n+1}-\hat{\boldsymbol{u}}^{n}+\nabla_{h}\left(\phi^{n}-\phi^{n+1}\right)=0, \quad \text { in } \Omega, \\
\nabla_{h} \cdot \boldsymbol{u}^{n+1}=0, \quad \text { at } x= \pm 1 . \\
\frac{\partial\left(\phi^{n+1}-\phi^{n}\right)}{\partial \boldsymbol{n}}=\boldsymbol{n} \cdot \boldsymbol{u}^{n+1}=0,
\end{array}\right.
\end{aligned}
$$

We note that (4.16) is almost a discrete version of (1.14), except for the appearance of $\mathcal{N}_{h}\left(\boldsymbol{u}^{n}, \boldsymbol{u}^{n}\right)$, a nonlinear term. 
Let $\boldsymbol{u}_{0}(\boldsymbol{x}, t), \phi_{0}(\boldsymbol{x}, t)$ be solutions of the decoupled system (4.10), (4.12), which are guaranteed by Lemma 4.2 and Remark 4.1 to be smooth in the sense that the divided differences of various orders are bounded.

Next, we define $\boldsymbol{u}_{1}(\boldsymbol{x}, t)$ as the solution of the following system

$$
\left\{\begin{array}{l}
\partial_{t} \boldsymbol{u}_{1}+\nabla_{h} p_{1}+\mathcal{N}_{h}\left(\boldsymbol{u}_{0}, \boldsymbol{u}_{1}\right)+\mathcal{N}_{h}\left(\boldsymbol{u}_{1}, \boldsymbol{u}_{0}\right) \\
=\Delta_{h} \boldsymbol{u}_{1}+\partial_{t} \Delta_{h} \boldsymbol{a}_{0}-\frac{1}{2} \partial_{t}^{2} \boldsymbol{a}_{0}, \quad \text { in } \Omega \\
\nabla_{h} \cdot \boldsymbol{u}_{1}=0, \quad \text { in } \Omega \\
\left.\boldsymbol{u}_{1}\right|_{x= \pm 1}=\left.\partial_{t} \nabla_{h} \phi_{0}\right|_{x= \pm 1},
\end{array}\right.
$$

with suitable initial data for $\boldsymbol{u}_{1}$, and let $\phi_{1}(\boldsymbol{x}, t)$ be the solution of the following discrete heat equation:

$$
\left\{\begin{array}{l}
\partial_{t} \phi_{1}-\Delta_{h} \phi_{1}+p_{1}=0, \quad \text { in } \Omega, \\
\frac{\partial \phi_{1}}{\partial \boldsymbol{n}}=0, \quad \text { on } \partial^{\prime} \Omega,
\end{array}\right.
$$

with suitable initial data for $\phi_{1}$. We know from Lemma 4.3 and Remark 4.2 that (4.17) has a smooth solution.

Let $\boldsymbol{u}_{2}(\boldsymbol{x}, t)$ be the solution of the (spatially) discrete Stokes equations with the prescribed boundary condition and some suitable initial data

$$
\left\{\begin{array}{l}
\partial_{t} \boldsymbol{u}_{2}+\nabla_{h} p_{2}=\triangle_{h} \boldsymbol{u}_{2}, \quad \text { in } \Omega, \\
\nabla_{h} \cdot \boldsymbol{u}_{2}=0, \quad \text { in } \Omega, \\
\left.\boldsymbol{u}_{2}\right|_{x= \pm 1}=\left.\left(\frac{1}{2} \partial_{t}^{2} \nabla_{h} \phi_{0}-\partial_{t} \boldsymbol{u}_{1}+\partial_{t} \nabla_{h} \phi_{1}\right)\right|_{x= \pm 1} .
\end{array}\right.
$$

Subsequently, we let

$$
\hat{\boldsymbol{u}}_{1}=\boldsymbol{u}_{1}+\partial_{t} \boldsymbol{a}_{0}
$$

and

$$
\hat{\boldsymbol{u}}_{2}=\boldsymbol{u}_{2}+\frac{1}{2} \partial_{t}^{2} \boldsymbol{a}_{0}+\partial_{t} \boldsymbol{u}_{1}-\partial_{t} \nabla_{h} \phi_{1}
$$

Now we construct

$$
\left\{\begin{array}{l}
U^{* n}=\boldsymbol{u}_{0}+\Delta t \hat{\boldsymbol{u}}_{1}+\Delta t^{2} \hat{\boldsymbol{u}}_{2} \\
U^{n}=\boldsymbol{u}_{0}+\triangle t \boldsymbol{u}_{1}+\triangle t^{2} \boldsymbol{u}_{2} \\
\Phi^{n}=\phi_{0}+\triangle t \phi_{1}
\end{array}\right.
$$

and substitute them into (4.16). Similar to the computations and arguments in the spatially continuous case and doing Taylor expansions of $\boldsymbol{u}_{h}$ and $\nabla_{h} \phi$ w.r.t. time $t$, we obtain

$$
\left\{\begin{array}{l}
\frac{U^{* n}-U^{n}}{\Delta t}+\mathcal{N}_{h}\left(U^{n}, U^{n}\right)+\Delta_{h} \nabla_{h} \Phi^{n}=\Delta_{h} U^{* n}+\Delta t^{2} \boldsymbol{f}^{n}, \quad \text { in } \Omega, \\
U^{* n}=0, \quad \text { at } x= \pm 1
\end{array}\right.
$$




$$
\left\{\begin{array}{l}
U^{n+1}-U^{* n}+\nabla_{h}\left(\Phi^{n}-\Phi^{n+1}\right)=\Delta t^{3} \boldsymbol{g}^{n}, \quad \text { in } \Omega, \\
\nabla_{h} \cdot U^{n+1}=0, \quad \text { in } \Omega, \\
\frac{\partial \Phi^{n+1}}{\partial \boldsymbol{n}}=\boldsymbol{n} \cdot U^{n+1}=0, \quad \text { at } x= \pm 1,
\end{array}\right.
$$

where $\boldsymbol{f}^{n}$ and $\boldsymbol{g}^{n}$ are bounded and smooth if $\left(\boldsymbol{u}_{0}, \phi_{0}\right)$ is sufficiently smooth. It can be seen that the only difference between (4.22) and (4.16) is the higher order truncation error terms $\triangle t^{2} \boldsymbol{f}^{n}, \triangle t^{3} \boldsymbol{g}^{n}$.

It is obvious that

$$
\max _{0 \leq t^{n} \leq T}\left\|U^{n}(\cdot)\right\|_{W^{1, \infty}} \leq C^{*}
$$

Under the compatibility condition

$$
\partial_{t} \nabla_{h} \phi_{0}(\boldsymbol{x}, 0)=0, \quad \text { on } \partial^{\prime} \Omega,
$$

we can choose

$$
\boldsymbol{u}_{1}(\boldsymbol{x}, 0)=0 .
$$

Then we have a second order approximate initial data

$$
U^{0}(\boldsymbol{x})=\boldsymbol{u}_{0}(\boldsymbol{x}, 0)+\triangle t^{2} \boldsymbol{w}^{0}(\boldsymbol{x}),
$$

where $\boldsymbol{w}^{0}$ is a bounded function.

4.2. Proof of Theorem 4.1. Assume a priori that

$$
\max _{0 \leq t^{n} \leq T}\left\|\boldsymbol{u}^{n}\right\|_{W^{1, \infty}} \leq \tilde{C} .
$$

In the following estimate, the constant will sometimes depend on $C^{*}$ and $\tilde{C}$. We define

$$
\boldsymbol{e}^{n}=U^{n}-\boldsymbol{u}^{n}, \quad \hat{\boldsymbol{e}}^{n}=U^{* n}-\hat{\boldsymbol{u}}^{n}, \quad q^{n}=\Phi^{n}-\phi^{n} .
$$

The following system of error equations is obtained by subtracting (4.22) from

$$
\left\{\begin{array}{l}
\frac{\hat{\boldsymbol{e}}^{n}-\boldsymbol{e}^{n}}{\Delta t}+\mathcal{N}_{h}\left(\boldsymbol{e}^{n}, U^{n}\right)+\mathcal{N}_{h}\left(\boldsymbol{u}^{n}, \boldsymbol{e}^{n}\right)+\nabla_{h} \triangle_{h} q^{n}=\Delta_{h} \hat{\boldsymbol{e}}^{n}+\triangle t^{2} \boldsymbol{f}^{n}, \quad \text { in } \Omega, \\
\hat{\boldsymbol{e}}^{n}=0, \quad \text { at } x= \pm 1
\end{array}\right.
$$

$$
\left\{\begin{array}{l}
\boldsymbol{e}^{n+1}-\hat{\boldsymbol{e}}^{n}+\nabla_{h}\left(q^{n}-q^{n+1}\right)=\Delta t^{3} \boldsymbol{g}^{n}, \quad \text { in } \Omega, \\
\nabla_{h} \cdot \boldsymbol{e}^{n+1}=0, \quad \text { in } \Omega, \\
\frac{\partial q^{n+1}}{\partial \boldsymbol{n}}=\boldsymbol{n} \cdot \boldsymbol{e}^{n=1}=0, \quad \text { at } x= \pm 1,
\end{array}\right.
$$

The system (4.29) is similar to the system of the error equations for the spatially continuous Stokes equations (3.18). At first glance, (4.29) is almost a discrete version of (3.18). Then most of the techniques used in Section 3 can be applied here. But there are also some differences: the appearance of nonlinear error terms $\mathcal{N}_{h}\left(\boldsymbol{e}^{n}, U^{n}\right)$ and $\mathcal{N}_{h}\left(\boldsymbol{u}^{n}, \boldsymbol{e}^{n}\right)$, and the local truncation error terms appearing in (4.29) are of higher order than those of (3.18). We make higher order expansions in 
the spatially discrete case so that we can establish the $W^{1, \infty}$ estimate for numerical $\boldsymbol{u}^{n}$. This estimate is needed for nonlinear error terms so that part (v) of Lemma 4.1 can be applied. Using higher order expansions as we did in the consistency analysis part, the only thing we need to do is to apply the a priori estimate (4.27).

Taking the inner product of the equation in $(4.29 \mathrm{a})$ with $2 \hat{\boldsymbol{e}}^{n}$, we obtain

$$
\begin{aligned}
\left\|\hat{\boldsymbol{e}}^{n}\right\|^{2}- & \left\|\boldsymbol{e}^{n}\right\|^{2}+\left\|\hat{\boldsymbol{e}}^{n}-\boldsymbol{e}^{n}\right\|^{2}-2 \Delta t\left\langle\hat{\boldsymbol{e}}^{n}, \Delta_{h} \hat{\boldsymbol{e}}^{n}\right\rangle \\
\leq & \Delta t^{5}\left\|\boldsymbol{f}^{n}\right\|^{2}+\Delta t\left\|\hat{\boldsymbol{e}}^{n}\right\|^{2}-2 \Delta t\left\langle\hat{\boldsymbol{e}}^{n}, \mathcal{N}_{h}\left(\boldsymbol{e}^{n}, U^{n}\right)\right\rangle \\
& -2 \triangle t\left\langle\hat{\boldsymbol{e}}^{n}, \mathcal{N}_{h}\left(\boldsymbol{u}^{n}, \boldsymbol{e}^{n}\right)\right\rangle-2 \Delta t\left\langle\hat{\boldsymbol{e}}^{n}, \nabla_{h} \Delta_{h} q^{n}\right\rangle .
\end{aligned}
$$

By Lemma 4.1, parts (iv) and (v), and the a priori estimate (4.27), we get

$$
\begin{gathered}
\left\|\hat{\boldsymbol{e}}^{n}\right\|^{2}-\left\|\boldsymbol{e}^{n}\right\|^{2}+\left\|\hat{\boldsymbol{e}}^{n}-\boldsymbol{e}^{n}\right\|^{2}+\Delta t\left\|\nabla_{h} \hat{\boldsymbol{e}}^{n}\right\|^{2}+\Delta t\left\|\nabla_{h} \cdot \hat{\boldsymbol{e}}^{n}\right\|^{2} \\
\leq \Delta t^{5}\left\|\boldsymbol{f}^{n}\right\|^{2}+C \triangle t\left(\left\|\hat{\boldsymbol{e}}^{n}\right\|^{2}+\left\|\boldsymbol{e}^{n}\right\|^{2}\right) \\
\quad+\frac{1}{2} \triangle t\left\|\nabla_{h} \hat{\boldsymbol{e}}^{n}\right\|^{2}-2 \triangle t\left\langle\hat{\boldsymbol{e}}^{n}, \nabla_{h} \Delta_{h} q^{n}\right\rangle
\end{gathered}
$$

Once again, as can be seen, to use Lemma $4.1(\mathrm{v})$, we must have an a priori estimate (4.27). This requires us to do second order expansions in the spatially discrete case.

By the triangle inequality for the discrete $L^{2}$ norm,

$$
\left\|\hat{e}^{n}\right\| \leq\left\|\hat{e}^{n}-e^{n}\right\|+\left\|e^{n}\right\|
$$

we have

$$
\begin{aligned}
& \left\|\hat{\boldsymbol{e}}^{n}\right\|^{2}-\left\|\boldsymbol{e}^{n}\right\|^{2}+\frac{7}{8}\left\|\hat{\boldsymbol{e}}^{n}-\boldsymbol{e}^{n}\right\|^{2}+\triangle t\left\|\nabla_{h} \hat{\boldsymbol{e}}^{n}\right\|^{2}+\triangle t\left\|\nabla_{h} \cdot \hat{\boldsymbol{e}}^{n}\right\|^{2} \\
& \leq \triangle t^{5}\left\|\boldsymbol{f}^{n}\right\|^{2}+C \triangle t\left\|\boldsymbol{e}^{n}\right\|^{2}+\frac{1}{2} \triangle t\left\|\nabla_{h} \hat{\boldsymbol{e}}^{n}\right\|^{2}-2 \triangle t\left\langle\hat{\boldsymbol{e}}^{n}, \nabla_{h} \Delta_{h} q^{n}\right\rangle .
\end{aligned}
$$

Taking the inner product of the first equation in (4.29b) with $2 e^{n+1}$ and applying Lemma 4.1(iii) yields

$$
\left\|\boldsymbol{e}^{n+1}\right\|^{2}-\left\|\hat{\boldsymbol{e}}^{n}\right\|^{2}+\left\|\boldsymbol{e}^{n+1}-\hat{\boldsymbol{e}}^{n}\right\|^{2} \leq \Delta t\left\|\boldsymbol{e}^{n+1}\right\|^{2}+\triangle t^{5}\left\|\boldsymbol{g}^{n}\right\|^{2} .
$$

Combining (4.33) and (4.34), we get

$$
\begin{aligned}
& \left\|\boldsymbol{e}^{n+1}\right\|^{2}-\left\|\boldsymbol{e}^{n}\right\|^{2}+\frac{7}{8}\left\|\hat{\boldsymbol{e}}^{n}-\boldsymbol{e}^{n}\right\|^{2}+\left\|\boldsymbol{e}^{n+1}-\hat{\boldsymbol{e}}^{n}\right\|^{2} \\
& \quad+\frac{1}{2} \triangle t\left\|\nabla_{h} \hat{\boldsymbol{e}}^{n}\right\|^{2}+\triangle t\left\|\nabla_{h} \cdot \hat{\boldsymbol{e}}^{n}\right\|^{2} \\
& \quad \leq \Delta t^{5}\left(\left\|\boldsymbol{f}^{n}\right\|^{2}+\left\|\boldsymbol{g}^{n}\right\|^{2}\right)+C \triangle t\left(\left\|\boldsymbol{e}^{n}\right\|^{2}+\left\|\boldsymbol{e}^{n+1}\right\|^{2}\right)-2 \triangle t\left\langle\hat{\boldsymbol{e}}^{n}, \nabla_{h} \Delta_{h} q^{n}\right\rangle .
\end{aligned}
$$

Estimating the last term in (4.35) is similar to (3.22):

$$
\begin{aligned}
I \equiv & -2 \Delta t\left\langle\hat{\boldsymbol{e}}^{n}, \nabla_{h} \Delta_{h} q^{n}\right\rangle \\
= & 2 \Delta t\left\langle\nabla_{h} \cdot \hat{\boldsymbol{e}}^{n}, \Delta_{h} q^{n}\right\rangle \\
= & -2 \Delta t\left\langle\Delta_{h}\left(q^{n+1}-q^{n}\right), \Delta_{h} q^{n}\right\rangle-2 \Delta t^{4}\left\langle\nabla_{h} \cdot \boldsymbol{g}^{n}, \Delta_{h} q^{n}\right\rangle \\
= & -\triangle t\left(\left\|\Delta_{h} q^{n+1}\right\|^{2}-\left\|\Delta_{h} q^{n}\right\|^{2}\right)+\triangle t\left\|\Delta_{h}\left(q^{n+1}-q^{n}\right)\right\|^{2} \\
& -2 \triangle t^{4}\left\langle\nabla_{h} \cdot \boldsymbol{g}^{n}, \Delta_{h} q^{n}\right\rangle \\
= & -\triangle t\left(\left\|\Delta_{h} q^{n+1}\right\|^{2}-\left\|\Delta_{h} q^{n}\right\|^{2}\right)+\triangle t\left\|\nabla_{h} \cdot \hat{\boldsymbol{e}}^{n}\right\|^{2}+\triangle t^{7}\left\|\boldsymbol{g}^{n}\right\|^{2} \\
& +2 \triangle t^{4}\left\langle\nabla_{h} \cdot \hat{\boldsymbol{e}}^{n}, \nabla_{h} \cdot \boldsymbol{g}^{n}\right\rangle-2 \triangle t^{4}\left\langle\nabla_{h} \cdot \boldsymbol{g}^{n}, \Delta_{h} q^{n}\right\rangle .
\end{aligned}
$$


Then (4.36) gives us

$$
\begin{aligned}
I \leq & -\triangle t\left(\left\|\Delta_{h} q^{n+1}\right\|^{2}-\left\|\Delta_{h} q^{n}\right\|^{2}\right)+\triangle t\left\|\nabla_{h} \cdot \hat{\boldsymbol{e}}^{n}\right\|^{2}+\triangle t^{2}\left\|\Delta_{h} q^{n}\right\|^{2} \\
& +2 \triangle t^{6}\left\|\nabla_{h} \cdot \boldsymbol{g}^{n}\right\|^{2}+\triangle t^{2}\left\|\nabla_{h} \cdot \hat{\boldsymbol{e}}^{n}\right\|^{2}+\triangle t^{7}\left\|\boldsymbol{g}^{n}\right\|^{2} .
\end{aligned}
$$

Going back to (4.35), we obtain

$$
\begin{aligned}
\left\|e^{n+1}\right\|^{2}-\left\|e^{n}\right\|^{2}+\frac{1}{2}\left\|\nabla_{h} \hat{\boldsymbol{e}}^{n}\right\|^{2}+\triangle t\left(\left\|\Delta_{h} q^{n+1}\right\|^{2}-\left\|\Delta_{h} q^{n}\right\|^{2}\right) \\
\leq C \triangle t\left(\left\|\boldsymbol{e}^{n}\right\|^{2}+\left\|\boldsymbol{e}^{n+1}\right\|^{2}\right)+\triangle t^{2}\left\|\Delta_{h} q^{n}\right\|^{2} \\
+C \triangle t^{5}\left(\left\|\boldsymbol{f}^{n}\right\|^{2}+\left\|\boldsymbol{g}^{n}\right\|^{2}+\triangle t\left\|\boldsymbol{g}^{n}\right\|_{H^{1}}^{2}\right)
\end{aligned}
$$

Grownwall's Lemma gives

$$
\left\|\boldsymbol{e}^{n}\right\|+\triangle t\left\|\nabla_{h} \hat{\boldsymbol{e}}^{n}\right\|+\triangle t^{1 / 2}\left\|\Delta_{h} q^{n}\right\| \leq C_{1} \triangle t^{2} .
$$

By the inverse inequality (4.4) we have

$$
\left\|\boldsymbol{e}^{n}\right\|_{L^{\infty}}+h\left\|\boldsymbol{e}^{n}\right\|_{W^{1, \infty}}+\triangle t^{1 / 2}\left\|\Delta_{h} q^{n}\right\|_{L^{\infty}} \leq C_{1} \frac{\triangle t^{2}}{h} .
$$

Under the CFL constraint

$$
\triangle t \leq \sqrt{\frac{1}{C_{1}}} \triangle x
$$

where $C_{1}$ only depends on the exact solution $\left(\boldsymbol{u}_{0}, \phi_{0}\right)$ and the a priori constant $\tilde{C}$ for the estimate of $\left\|\boldsymbol{u}^{n}\right\|_{W^{1, \infty}}$ in (4.27), we have

$$
\left\|\boldsymbol{e}^{n+1}\right\|_{L^{\infty}} \leq \sqrt{C_{1}} \triangle t, \quad\left\|\boldsymbol{e}^{n+1}\right\|_{W^{1, \infty}} \leq 1 .
$$

Therefore in (4.27) we can choose

$$
\tilde{C}=1+\max _{n \leq\left[\frac{T}{\Delta t}\right]}\left\|U^{n}(\cdot)\right\|_{W^{1, \infty}}
$$

so that $\tilde{C}$ depends only on the exact solution $\left(\boldsymbol{u}_{0}, \phi_{0}\right)$. This gives

$$
\left\|\boldsymbol{u}_{0}-\boldsymbol{u}_{h}\right\|_{L^{\infty}} \leq C \triangle t .
$$

By Lemma 4.2, we have

$$
\left\|\boldsymbol{u}-\boldsymbol{u}_{h}\right\|_{L^{\infty}} \leq C\left(\triangle t+h^{2}\right) .
$$

This completes the proof of Theorem 4.1.

\section{ANALYSis AND ERROR ESTIMATE OF THE Dirichlet GaUge FORMULATION}

Finally we look at the gauge method with Dirichlet boundary condition for $\phi$. For simplicity, we will concentrate on the spatially continuous case for Stokes equations:

$$
\left\{\begin{array}{l}
\frac{\boldsymbol{a}^{n+1}-\boldsymbol{a}^{n}}{\triangle t}=\triangle \boldsymbol{a}^{n+1}, \quad \text { in } \Omega, \\
\boldsymbol{a}^{n+1} \cdot \boldsymbol{n}=\frac{\partial \phi^{n}}{\partial \boldsymbol{n}}, \quad \boldsymbol{a}^{n+1} \cdot \boldsymbol{\tau}=0, \quad \text { on } \partial \Omega,
\end{array}\right.
$$


and

$$
\begin{aligned}
& \begin{cases}\triangle \phi^{n+1}=-\nabla \cdot \boldsymbol{a}^{n+1}, & \text { in } \Omega, \\
\phi^{n+1}=0, & \text { on } \partial \Omega,\end{cases} \\
& \boldsymbol{u}^{n+1}=\boldsymbol{a}^{n+1}+\nabla \phi^{n+1} .
\end{aligned}
$$

Next we state our theorem for the Dirichlet gauge formulation:

Theorem 5.1. Let $(\boldsymbol{u}, \phi)$ be a smooth solution of Stokes equation (2.1) with smooth initial data $\boldsymbol{u}^{0}(\boldsymbol{x})$, and let $\left(\boldsymbol{u}_{\triangle t}, \phi_{\triangle t}\right)$ be the numerical solution for the semi-discrete gauge method with Dirichlet boundary condition for the gauge variable (5.1)-(5.3). Then

$$
\left\|\boldsymbol{u}-\boldsymbol{u}_{\triangle t}\right\|_{L^{\infty}\left(0, T ; L^{2}\right)} \leq C \sqrt{\triangle t} .
$$

Proof. The analysis carried out in Section 2 can be applied to this formulation similarly. First we make a transformation. If we introduce $\hat{\boldsymbol{u}}^{n}=\boldsymbol{a}^{n+1}+\nabla \phi^{n}$, (5.1)-(5.3) can also be reformulated as

$$
\begin{gathered}
\left\{\begin{array}{l}
\frac{\hat{\boldsymbol{u}}^{n}-\boldsymbol{u}^{n}}{\Delta t}+\Delta \nabla \phi^{n}=\Delta \hat{\boldsymbol{u}}^{n}, \quad \text { in } \Omega, \\
\hat{\boldsymbol{u}}^{n}=0, \quad \text { on } \partial \Omega,
\end{array}\right. \\
\left\{\begin{array}{l}
\boldsymbol{u}^{n+1}-\hat{\boldsymbol{u}}^{n}+\nabla\left(\phi^{n}-\phi^{n+1}\right)=0, \quad \text { in } \Omega, \\
\nabla \cdot \boldsymbol{u}^{n+1}=0, \quad \text { in } \Omega, \\
\left(\phi^{n}-\phi^{n+1}\right)=0, \quad \text { on } \partial \Omega .
\end{array}\right.
\end{gathered}
$$

Note that (5.5) is the same as (1.14) except for the boundary condition for $\phi$.

We will repeat the procedure of Section 2. Let $\boldsymbol{u}_{\mathrm{e}}(\boldsymbol{x}, t), p_{\mathrm{e}}(\boldsymbol{x}, t)$ be the exact solution of the Stokes equations

$$
\left\{\begin{array}{l}
\partial_{t} \boldsymbol{u}_{\mathrm{e}}+\nabla p_{\mathrm{e}}=\Delta \boldsymbol{u}_{\mathrm{e}}, \quad \text { in } \Omega, \\
\nabla \cdot \boldsymbol{u}_{\mathrm{e}}=0, \quad \text { in } \Omega, \\
\boldsymbol{u}_{\mathrm{e}}=0, \quad \text { on } \partial \Omega,
\end{array}\right.
$$

and let $\phi_{\mathrm{e}}(\boldsymbol{x}, t)$ be a solution of the following heat equation with Dirichlet boundary condition:

$$
\left\{\begin{array}{l}
\partial_{t} \phi_{\mathrm{e}}=\Delta \phi_{\mathrm{e}}-p_{\mathrm{e}}, \quad \text { in } \Omega, \\
\phi_{\mathrm{e}}=0, \quad \text { on } \partial \Omega .
\end{array}\right.
$$

However, there is some trouble when we try to construct $\boldsymbol{u}_{1}$ in the expansion of the numerical scheme. As can be seen, (3.5) does not necessarily have a solution in the Dirichlet gauge formulation. Since $\partial_{t} \nabla \phi_{0}$ is not orthogonal to the normal vector at the boundary, this leads to the incompatibility of the boundary condition for $\boldsymbol{u}_{1}$. Yet, to continue our analysis, we can still construct an arbitrary field $\boldsymbol{u}_{1}$ such that

$$
\boldsymbol{u}_{1}=\partial_{t} \nabla \phi_{\mathrm{e}}, \quad \text { on } \partial \Omega \text {. }
$$


We still adopt the notation in Section 2. Let $\hat{\boldsymbol{u}}_{1}=\boldsymbol{u}_{1}+\partial_{t} \boldsymbol{a}_{\mathrm{e}}$, and construct

$$
U^{*}=\boldsymbol{u}_{\mathrm{e}}+\Delta t \hat{\boldsymbol{u}}_{1}, \quad U=\boldsymbol{u}_{\mathrm{e}}+\Delta t \boldsymbol{u}_{1}, \quad \Phi=\phi_{\mathrm{e}} .
$$

It must be mentioned here that $U$ is not divergence free up to an order $O(\triangle t)$ :

$$
\nabla \cdot U=\triangle t \boldsymbol{h}, \quad \text { where } \boldsymbol{h}=\nabla \cdot \boldsymbol{u}_{1} .
$$

This fact will reduce a $\sqrt{\triangle t}$ factor in our estimate, as we can see later. Using similar arguments as in Lemma 3.1, we have the following system analogous to (3.9):

$$
\begin{aligned}
& \left\{\begin{array}{l}
\frac{U^{* n}-U^{n}}{\Delta t}+\Delta \nabla \Phi^{n}=\Delta U^{* n}+\Delta t \boldsymbol{f}^{n}, \quad \text { in } \Omega, \\
U^{* n}=0, \quad \text { on } \partial \Omega,
\end{array}\right. \\
& \left\{\begin{array}{l}
U^{n+1}-U^{* n}+\nabla\left(\Phi^{n}-\Phi^{n+1}\right)=\Delta t^{2} \boldsymbol{g}^{n}, \quad \text { in } \Omega, \\
\nabla \cdot U^{n+1}=\Delta t \boldsymbol{h}^{n+1}, \quad \text { in } \Omega, \\
\Phi^{n}-\Phi^{n+1}=0, \quad \text { on } \partial \Omega, \\
U^{0}=\boldsymbol{u}^{0}+\Delta t \boldsymbol{w}^{0}, \quad \text { in } \Omega,
\end{array}\right.
\end{aligned}
$$

where $\boldsymbol{f}^{n}, \boldsymbol{g}^{n}, \boldsymbol{h}^{n+1}$ and $\boldsymbol{w}^{0}$ are bounded functions.

Using the same notation as in (3.17), i.e.

$$
\boldsymbol{e}^{n}=U^{n}-\boldsymbol{u}^{n}, \quad \hat{\boldsymbol{e}}^{n}=U^{* n}-\hat{\boldsymbol{u}}^{n}, \quad q^{n}=\Phi^{n}-\phi^{n},
$$

and subtracting (5.10) from (5.5), we get the system of error equations

$$
\begin{aligned}
& \left\{\begin{array}{l}
\frac{\hat{\boldsymbol{e}}^{n}-\boldsymbol{e}^{n}}{\Delta t}=\Delta \hat{\boldsymbol{e}}^{n}-\nabla \Delta q^{n}+\Delta t \boldsymbol{f}^{n}, \quad \text { in } \Omega, \\
\hat{\boldsymbol{e}}^{n}=0, \quad \text { on } \partial \Omega,
\end{array}\right. \\
& \left\{\begin{array}{l}
\boldsymbol{e}^{n+1}-\hat{\boldsymbol{e}}^{n}+\nabla\left(q^{n}-q^{n+1}\right)=\Delta t^{2} \boldsymbol{g}^{n}, \quad \text { in } \Omega, \\
\nabla \cdot \boldsymbol{e}^{n+1}=\Delta t \boldsymbol{h}^{n+1}, \quad \text { in } \Omega, \\
q^{n}-q^{n+1}=0, \quad \text { on } \partial \Omega \\
\boldsymbol{e}^{0}=\Delta t \boldsymbol{w}^{0}, \quad \text { in } \Omega .
\end{array}\right.
\end{aligned}
$$

We will continue to do energy estimates as in Section 2. Applying the same procedure, taking the inner product of the first equation of $(5.12 \mathrm{a})$ with $2 \hat{\boldsymbol{e}}^{n}$, we get

$$
\begin{aligned}
& \left\|\hat{\boldsymbol{e}}^{n}\right\|^{2}-\left\|\boldsymbol{e}^{n}\right\|^{2}+\left\|\hat{\boldsymbol{e}}^{n}-\boldsymbol{e}^{n}\right\|^{2}+2 \Delta t\left\|\nabla \hat{\boldsymbol{e}}^{n}\right\|^{2} \\
& \quad \leq \Delta t^{3}\left\|\boldsymbol{f}^{n}\right\|^{2}+C \triangle t\left\|\boldsymbol{e}^{n}\right\|^{2}-2 \triangle t \int_{\Omega} \hat{\boldsymbol{e}}^{n} \cdot \nabla \Delta q^{n} d \boldsymbol{x} .
\end{aligned}
$$

Taking the inner product of the equation of $(5.12 \mathrm{~b})$ with $2 \boldsymbol{e}^{n+1}$ yields

$$
\begin{aligned}
& \left\|\boldsymbol{e}^{n+1}\right\|^{2}-\left\|\hat{\boldsymbol{e}}^{n}\right\|^{2}+\left\|\boldsymbol{e}^{n+1}-\hat{\boldsymbol{e}}^{n}\right\|^{2} \\
& \quad \leq \Delta t\left\|\boldsymbol{e}^{n+1}\right\|^{2}+\triangle t^{3}\left\|\boldsymbol{g}^{n}\right\|^{2}-2 \int_{\Omega} \boldsymbol{e}^{n+1} \cdot \nabla\left(q^{n}-q^{n+1}\right) d \boldsymbol{x} .
\end{aligned}
$$


Next, we estimate the last term of the right hand side, which is caused by the fact that $U^{n}$ is not divergence-free:

$$
\begin{aligned}
I_{1} & \equiv-2 \int_{\Omega} \boldsymbol{e}^{n+1} \cdot \nabla\left(q^{n}-q^{n+1}\right) d \boldsymbol{x}=2 \int_{\Omega}\left(\nabla \cdot \boldsymbol{e}^{n+1}\right)\left(q^{n}-q^{n+1}\right) d \boldsymbol{x} \\
& =2 \triangle t \int_{\Omega} \boldsymbol{h}^{n+1}\left(q^{n}-q^{n+1}\right) d \boldsymbol{x} \leq C \triangle t^{2}\left\|\boldsymbol{h}^{n+1}\right\|^{2}+C_{1}\left\|q^{n}-q^{n+1}\right\|^{2} .
\end{aligned}
$$

Since $q^{n}-q^{n+1}=0$ on the boundary, by the Poincaré inequality we have

$$
\begin{aligned}
\left\|q^{n}-q^{n+1}\right\|^{2} & \leq C_{2}\left\|\nabla\left(q^{n}-q^{n+1}\right)\right\|^{2} \\
& \leq C_{2}\left\|\left(\boldsymbol{e}^{n+1}-\hat{\boldsymbol{e}}^{n}\right)+\triangle t^{2} \boldsymbol{g}^{n}\right\|^{2} \\
& \leq \frac{3}{2} C_{2}\left\|\boldsymbol{e}^{n+1}-\hat{\boldsymbol{e}}^{n}\right\|^{2}+C \triangle t^{4}\left\|\boldsymbol{g}^{n}\right\|^{2},
\end{aligned}
$$

Since we can always adjust $C_{1}$ so that $C_{1} C_{2} \leq \frac{1}{3}$, we have from (5.14)-(5.16) that

$$
\left\|\boldsymbol{e}^{n+1}\right\|^{2}-\left\|\hat{\boldsymbol{e}}^{n}\right\|^{2}+\frac{1}{2}\left\|\boldsymbol{e}^{n+1}-\hat{\boldsymbol{e}}^{n}\right\|^{2} \leq \triangle t\left\|\boldsymbol{e}^{n+1}\right\|^{2}+C \triangle t^{2}\left\|\boldsymbol{h}^{n+1}\right\|^{2}+\triangle t^{3}\left\|\boldsymbol{g}^{n}\right\|^{2} .
$$

The estimates (3.22) and (3.23) are still valid here. Finally we obtain

$$
\begin{aligned}
\left\|\boldsymbol{e}^{n+1}\right\|^{2}-\left\|\boldsymbol{e}^{n}\right\|^{2}+\frac{1}{2} \triangle t\left\|\nabla \hat{\boldsymbol{e}}^{n}\right\|^{2}+\triangle t\left(\left\|\Delta q^{n+1}\right\|^{2}-\left\|\Delta q^{n}\right\|^{2}\right) \\
\leq C \triangle t\left(\left\|\boldsymbol{e}^{n}\right\|^{2}+\left\|\boldsymbol{e}^{n+1}\right\|^{2}\right)+\triangle t^{2}\left\|\triangle q^{n}\right\|^{2} \\
\quad+C \triangle t^{2}\left\|\boldsymbol{h}^{n+1}\right\|^{2}+C \triangle t^{3}\left(\left\|\boldsymbol{f}^{n}\right\|^{2}+\left\|\boldsymbol{g}^{n}\right\|^{2}+\triangle t\left\|\boldsymbol{g}^{n}\right\|_{H^{1}}^{2}\right) .
\end{aligned}
$$

Applying the discrete Grownwall lemma to the last inequality, we arrive at

$$
\left\|\boldsymbol{e}^{n}\right\|+\triangle t^{1 / 2}\left\|\nabla \hat{\boldsymbol{e}}^{n}\right\|+\triangle t^{1 / 2}\left\|\Delta q^{n}\right\| \leq C \sqrt{\triangle t},
$$

where only the $\sqrt{\triangle t}$ error estimate for the velocity field is available. Theorem 5.1 is now proven.

\section{ACKNOWLEDGMENT}

We thank Weinan E for many insightful discussions.

\section{REFERENCES}

1. A.S. Almgren, J.B. Bell, and W.G. Szymczak, A numerical method for the incompressible Navier-Stokes equations based on an approximate projection, SIAM J. Sci. Comput. $\mathbf{1 7}$ (1996), 358-369. MR 96j:76104

2. A.J. Chorin, Numerical solution of the Navier-Stokes equations, Math. Comp. 22 (1968), 745-762. MR 39:3723

3. J.B. Bell, P. Colella, and H.M. Glaz, A second order projection method for the incompressible Navier-Stokes equations, J. Comput. Phys. 85 (1989), 257-283. MR 90i:76002

4. T. Buttke, Velicity methods: Lagrangian numerical methods which preserve the Hamiltonian structure of incompressible fluid flow, Vortex Flows and Related Numerical Methods, (Grenoble, 1992; J. T. Beale et al., eds.), NATO Adv. Sci. Inst. Ser. C: Math. Phys. Sci., vol. 395, Kluwer, Dordrecht, 1993, pp. 39-57. MR 94m:760093

5. T. Buttke and A. Chorin, Turbulence calculation using magnetization variables, Appl. Numer. Math. 12 (1993), 47-54. MR 94e:76016

6. Weinan E and J.-G. Liu, Gauge method for viscous incompressible flows, submitted to J. Comp. Phys. (1996) 
7. Weinan E and J.-G. Liu, Gauge finite element method for incompressible flows, to appear in Int. J. Num. Meth. Fluids (2000).

8. Weinan E and J.-G. Liu, Projection method I: Convergence and numerical boundary layers, SIAM J. Numer. Anal. 32 (1995), 1017-1057. Projection method III: Spatial discretizations on the staggered grid, to appear Math. Comp. MR 96e:65061

9. Weinan E and J.-G. Liu, Finite difference schemes for incompressible flows in the impulsevelocity formulation, J. Comput. Phys. 130 (1997), 67-76. MR 97j:76036

10. P.M. Gresho and R.L. Sani, On pressure boundary conditions for the incompressible NavierStokes equations, Int. J. Numer. Methods Fluids 7 (1987), 1111-1145.

11. G. E. Karniadakis and S. J. Sherwin, Spectral/hp Element Methods for CFD, Oxford University Press, New York (1999)

12. J. Kim and P. Moin, Application of a fractional-step method to incompressible Navier-Stokes equations, J. Comp. Phys. 59 (1985), 308-323. MR 87a:76046

13. J.H. Maddocks and R.L. Pego, An unconstrained Hamiltonian formulation for incompressible fluid, Comm. Math. Phys. 170 (1995), 207-217. MR 96a:76085

14. S.A. Orszag, M. Israeli, and M.O. Deville, Boundary conditions for incompressible flows, J. Scientific Computing 1 (1986), 75-111.

15. V.I. Oseledets, On a new way of writing the Navier-Stokes equation. The Hamiltonian formulation, J. Russ. Math. Surveys 44 (1989), 210-211. MR 91e:58173

16. G. Russo and P. Smereka, Impulse formulation of the Euler equations: general properties and numerical methods, J. Fluid Mech. 391 (1999), 189-209. CMP 99:17

17. J. Shen, On error estimates of projection methods for Navier-Stokes equation: first order schemes, SIAM J. Numer. Anal. 29 (1992), 57-77. MR 92m:35213

18. J. Shen, On error estimates of some higher order projection and penalty-projection methods for Navier-Stokes equations, Numer. Math. 62 (1992), 49-73. MR 93a:35122

19. G. Strang, Accurate partial differential methods II. Non-linear problems, Numer. Math. 6 (1964), 37-46. MR 29:4215

20. R. Temam, Sur l'approximation de la solution des equation de Navier-Stokes par la méthode des fractionnaires II, Arch. Rational Mech. Anal. 33 (1969), 377-385. MR 39:5968

21. J. van Kan, A second order accurate pressure correction scheme for viscous incompressible flow, SIAM J. Sci. Comput. 7 (1986), 870-891. MR 87h:76008

22. B. R. Wetton, Error analysis for Chorin's original fully discrete projection method and regularizations in space and time, SIAM J. Numer. Anal. 34 (1997), 1683-1697. MR 95c:65161

Institute for Physical Science and Technology and Department of Mathematics, University of Maryland, College Park, Maryland 20742

Current address: Department of Mathematics, Indiana University, Bloomington, Indiana 47405

E-mail address: cwang@math.umd.edu

Institute for Physical Science and Technology and Department of Mathematics, University of Maryland, College Park, Maryland 20742

E-mail address: jliu@math.umd.edu 